\title{
Some Recent Experimental Results from Fermilab
}

\author{
H.E. Montgomery \\ Fermi National Accelerator Laboratory \\ P.O. Box 500, Batavia, Illinois 60510
}

February 1994

Presented at the International School on Sub-nuclear Physics, Erice, Trapani, Sicily, Italy, July 1993 


\section{Disclaimer}

This report was prepared as an account of work sponsored by an agency of the United States Government. Neither the United States Government nor any agency thereof, nor any of their employees, makes any warranty, express or implied, or assumes any legal liability or responsibility for the accuracy, completeness, or usefulness of any information, apparatus, product, or process disclosed, or represents that its use would not infringe privately owned rights. Reference herein to any specific commercial product, process, or service by trade name, trademark, manufacturer, or otherwise, does not necessarily constitute or imply its endorsement, recommendation, or favoring by the United States Government or any agency thereof. The views and opinions of authors expressed herein do not necessarily state or reflect those of the United States Government or any agency thereof. 


\title{
Some Recent Experimental Results from Fermilab
}

\author{
H.E.Montgomery \\ Fermilab \\ P.O.Box 500 \\ Batavia, IL 60510 \\ February 15, 1994
}

\begin{abstract}
A selection of recent experimental results from experiments at Fermilab is presented and discussed. In some cases the information is updated from that available at the time of the presentation of the talk.
\end{abstract}

Lecture given at the International School of Sub-nuclear Physics, Erice, Trapani, Sicily, Italy, July, 1993. 


\section{Introduction, Accelerator, Experiments.}

This lecture in contrast to many of the others at this school is a presentation of data. The Tevatron accelerator at Fermilab has been running for physics in both fixed target and collider modes, most recently the latter. The breadth of the results from the different experiments is very impressive and in order to give this talk I have been forced to be selective. Nevertheless, I have chosen to give many samples rather than just a "ew results with in depth discussion. In this way it is hoped that an impression of the potential and the contributions to the advancement of the science are given.

In 1992-3 the Tevatron operated in collider mode with $900 \mathrm{GeV}$ anti-protons and $900 \mathrm{GeV}$ protons. The accelerator surpassed many records for anti-proton production and for luminosity, exceeding $910^{30} \mathrm{~cm}^{-2} . \mathrm{sec}^{-1}$ in peak luminosity. The integrated luminosity delivered to the experiments was more than $30 \mathrm{pb}^{-1}, \mathrm{CDF}$ was able to log data corresponding to $21 \mathrm{pb}^{-1}$ and $\mathrm{D} \emptyset$, the new Tevatron collider experiment, $16 \mathrm{pb}^{-1}$. The difference between the two is largely accounted for by the fact that the conventional Main Ring accelerator, which is used to produce anti-protons, actually passes through the outer part of the DØ calorimeter. Thus far this has been handled primarily by gating off the experiment when beam in the Main Ring is passing.

While the talk will cover many experiments I would like to illustrate the actual apparati with just four examples. First, in Fig 1, the E687, photoproduction experimental apparatus is shown. This is typical of the " $800 \mathrm{GeV}$ " program fixed target experiments. It is many tens of metres long and as in all cases where the study of heavy quarks is concerned there is an elaborate silicon micro-strip detector, which has sufficient tracking precision to measure the secondary vertices associated with the decays of charm and bottom particles.

A contrast is offered by the E760 apparatus, Fig 2., which is installed in the anti-proton accumulator where it uses low energy anti-protons and a hydrogen gas jet to study charmonium states in formation. The technique evades the restriction in quantum numbers associated with electron positron formation experiments and bore fruit with the first observation[1] of the ${ }^{1} P_{1}$, $c \bar{c}$ state a year or more ago. Given the low energy, the apparatus is compact, but the asymmetric kinematics mean it is somewhat different from a low energy collider experiment.

The largest experiments at Fermilab are the two Tevatron collider experiments, D $\emptyset$, shown in Fig. 3. and CDF in Fig. 4. $D \emptyset$ is new, it operated for the first time in the 1992-3 collider run. It has a hermetic calorimeter, good muon coverage and a compact non-magnetic central tracking system. In contrast, CDF has magnetic tracking and the new feature of the detector for the recent run was the addition of a $\mathbf{4 5 0 0 0}$ channel silicon microstrip barrel. This introduced precision determination of heavy quark decay vertices to high energy hadron colliders. 


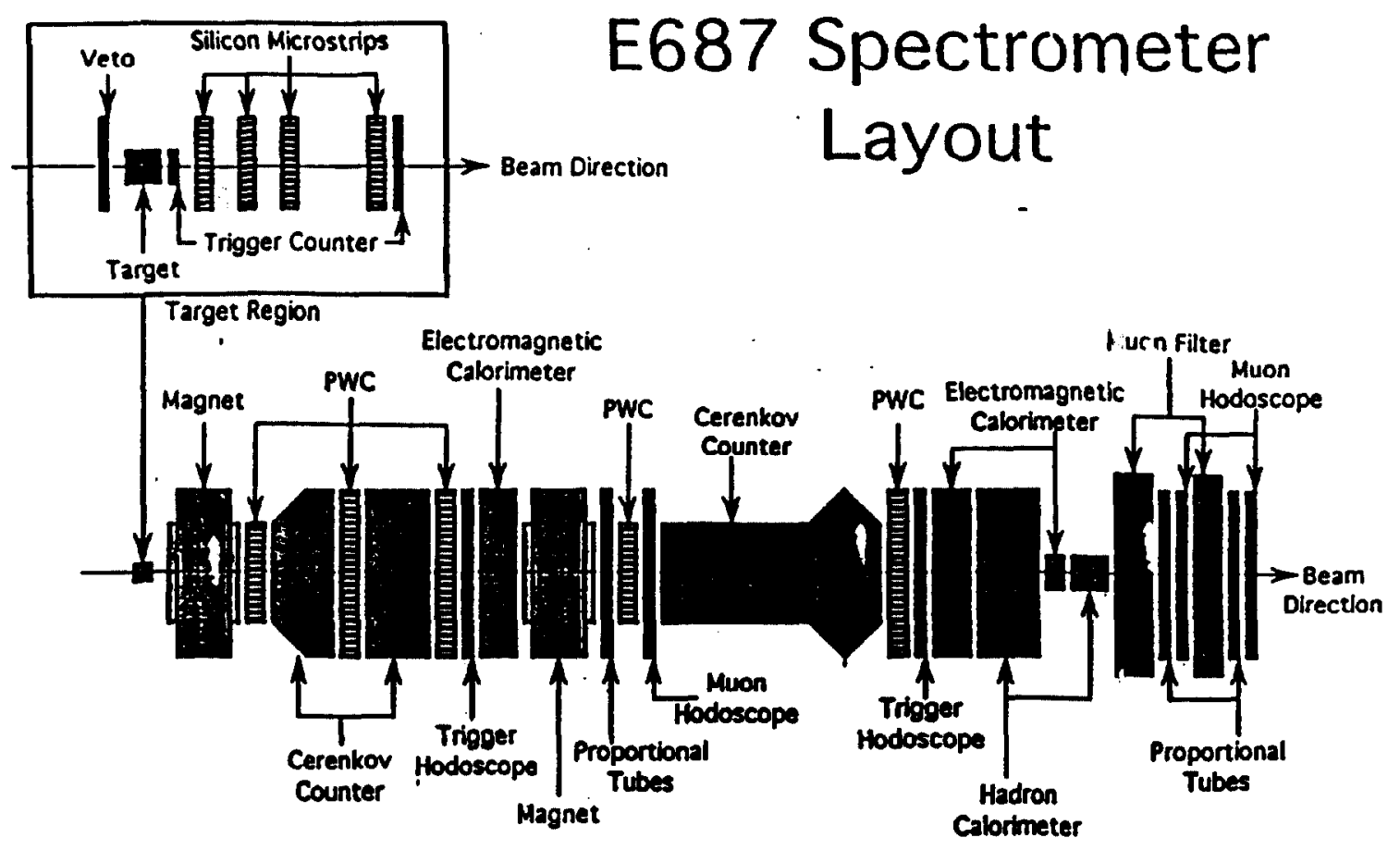

Figure 1: The E687 Apparatus.

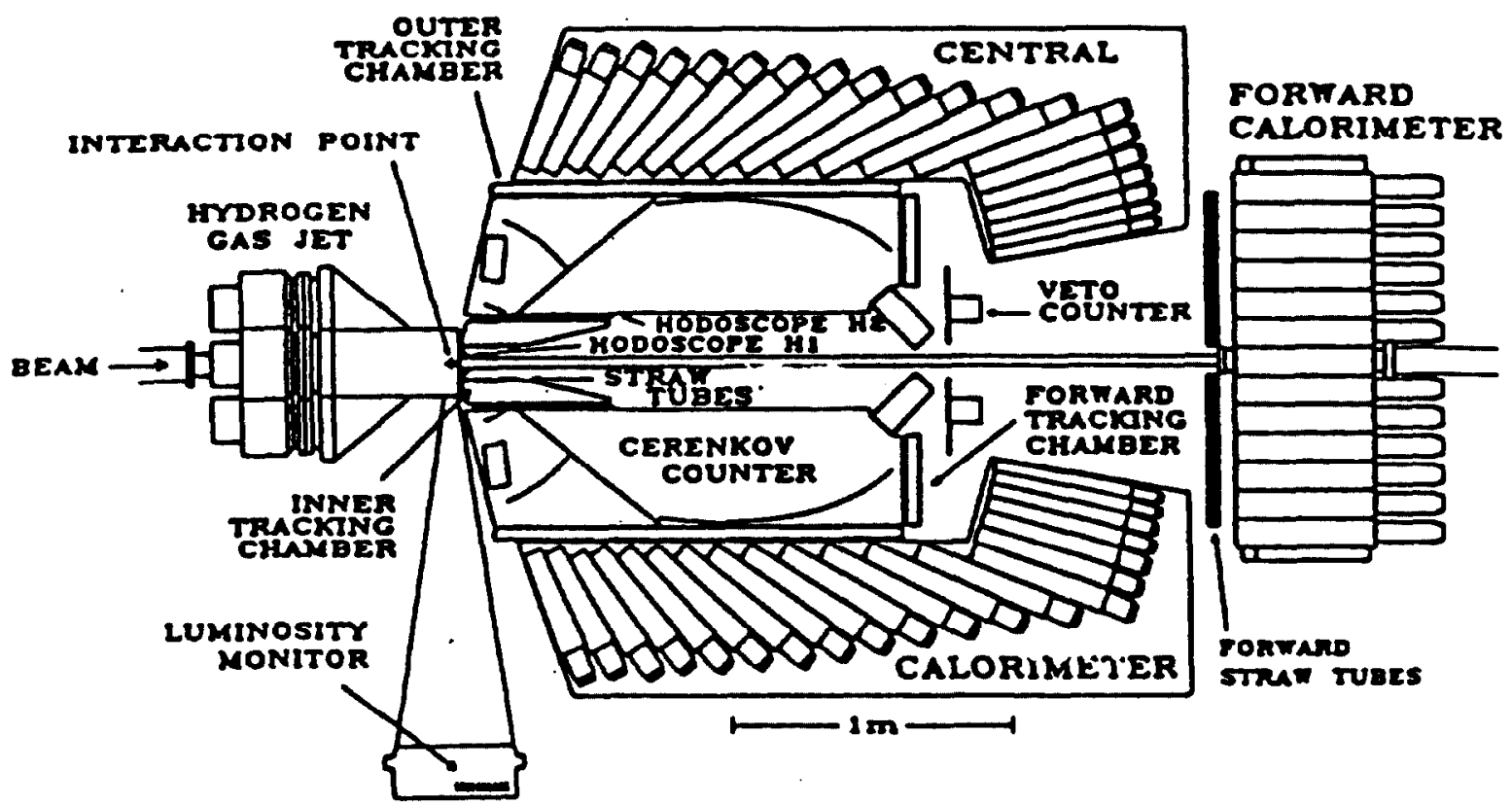

Figure 2: The E760 Apparatus. 


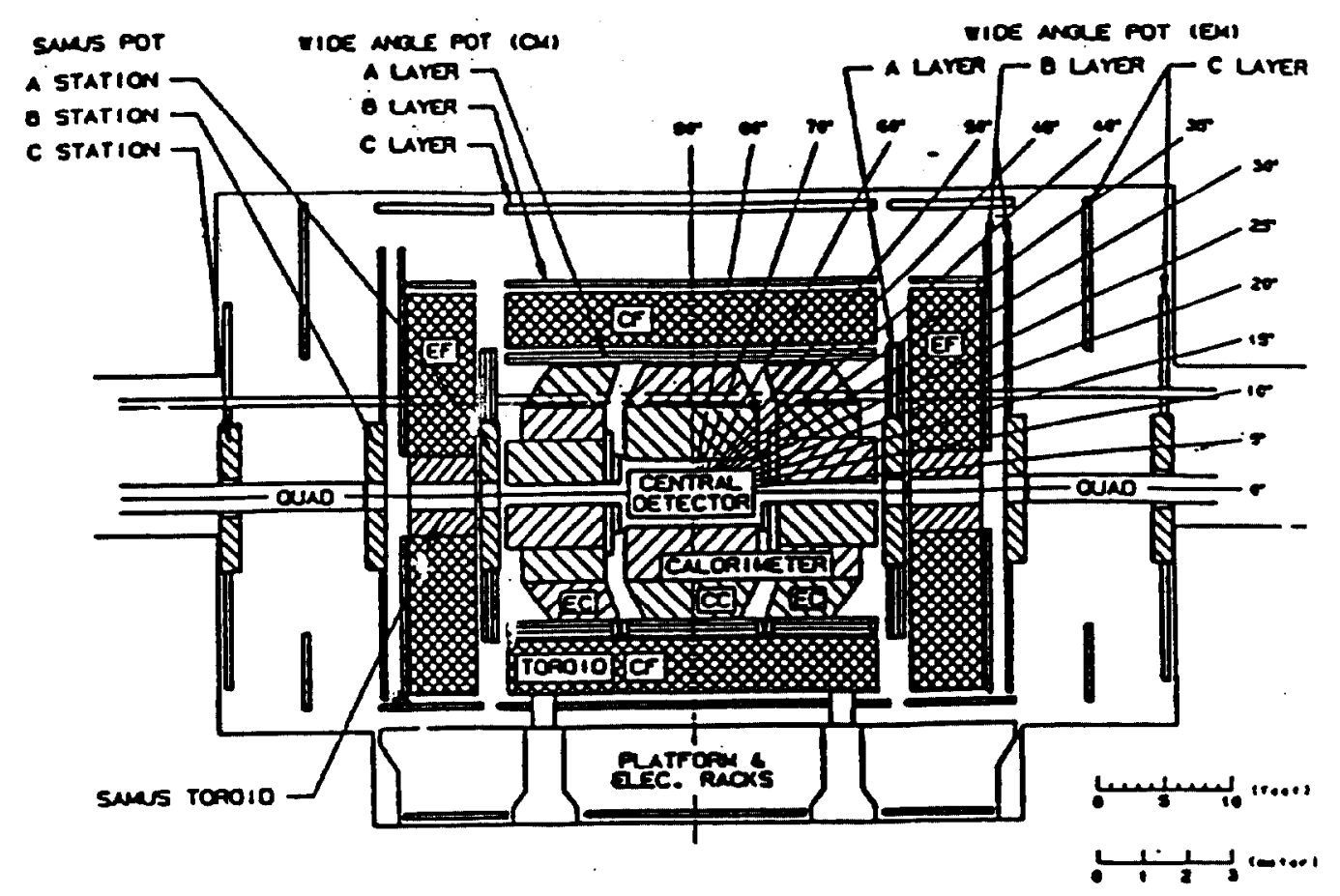

Figure 3: The D $\emptyset$ Experiment Apparatus.

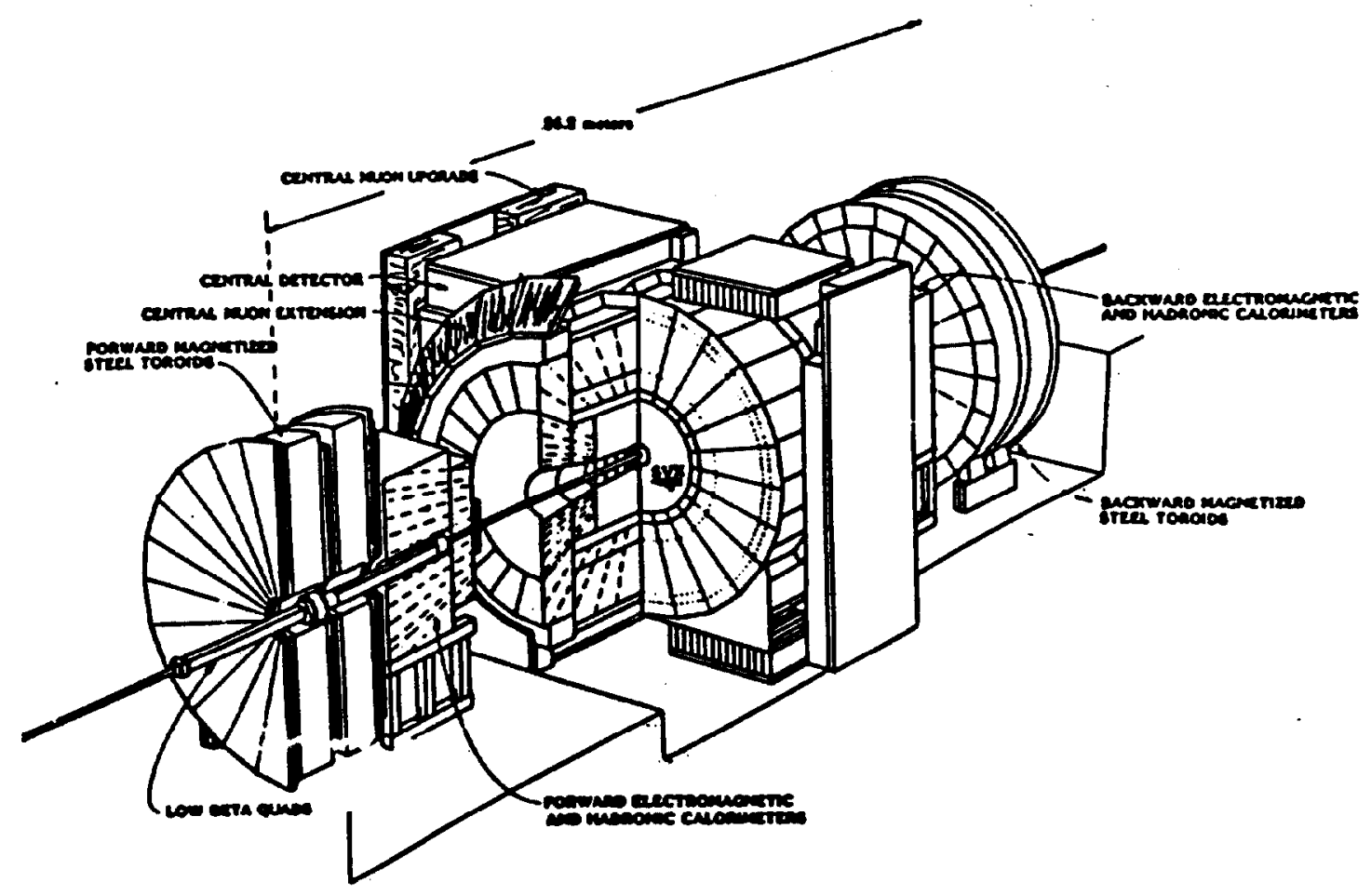

Figure 4: The CDF Experiment Apparatus. 


\section{Cross-Sections, QCD Measurements}

Because of the possibility that in certain circumstances they can interact directly with partons, photons figure prominently as probes of the strong interaction.

Real photon interactions contain both soft interactions, where the photon behaves as a hadronic vector meson, and hard interactions involving the photon and individual partons. vets are produced through a QCD Compton process and through the photon gluon fusion graph. In addition, there may be higher order resolved photon process. Typically these components lead to one, two and three jets respectively, in addition to the target recoil jet. The simplest parton diagrams produce no beam spectator jet, whereas the resolved photon process coes. The expected topology with two recoiling high $p_{2}$ jets is observed by E683[2], working

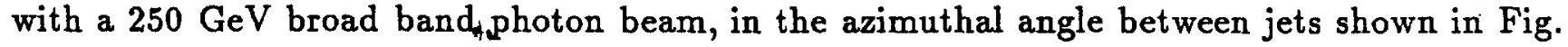
5a. The forward calorimeter energy distribution shown in Fig. $5 b$ is well described by the expected proportions of these different processes.

Using the transverse energy distribution of resolved forward jets in deep inelastic scattering of muons, E665 has determined[3] the strong coupling constant, $\alpha_{s}$, see Fig 6a. As with real photons there are kinematic regions where muon scattering exhibits a hadron like component of the virtual photon. Studies[4] of the hadronic final state in this shadowing region have so far shed little light on the question of which component of the final state is being shadowed. E665 has extended its jet studies to nuclear targets and they show preliminary evidence[5] that the muli-jet final states are more shadowed than the single parton jet final states. Figure $6 \mathrm{~b}$ ) shows the ratio of yields of events of different topologies compared to that for the elementary deuteron target as a function of $x_{B j} . x_{B j}<0.01$ is where shadowing is observed in the total virtual photon cross-section in the same data[6].

Single photons in the final state can be produced through QCD compton scattering, quarkantiquark annihilation and quark bremsstrahlung. The challenge experimentally is to distinguish single photons from the decay photons from $\pi^{0} \mathrm{~s}$. E706 working with a $530 \mathrm{GeV}$ beam has measured[7] single photon cross-sections as a function of $p_{T}$ for incident pions and protons. The results are shown in Fig 7. The data can be described by next to leading order QCD calculations and are of sufficient precision to distinguisis different parton distribution sets. However, at next to leading order the predictions still show a dependence on the definition of scale. The collider experiments are attempting to extend their measurements to large $\eta$. The sensitivity to the calculations then extends into low effective $x_{B j}$, where the calculations are not well tested. Figure 8 shows the ratio between the measured[8] yields in two different ranges of $\eta$ from CDF. It appears as though the theoretical calculations have the wrong trend at low $p_{T}$. 

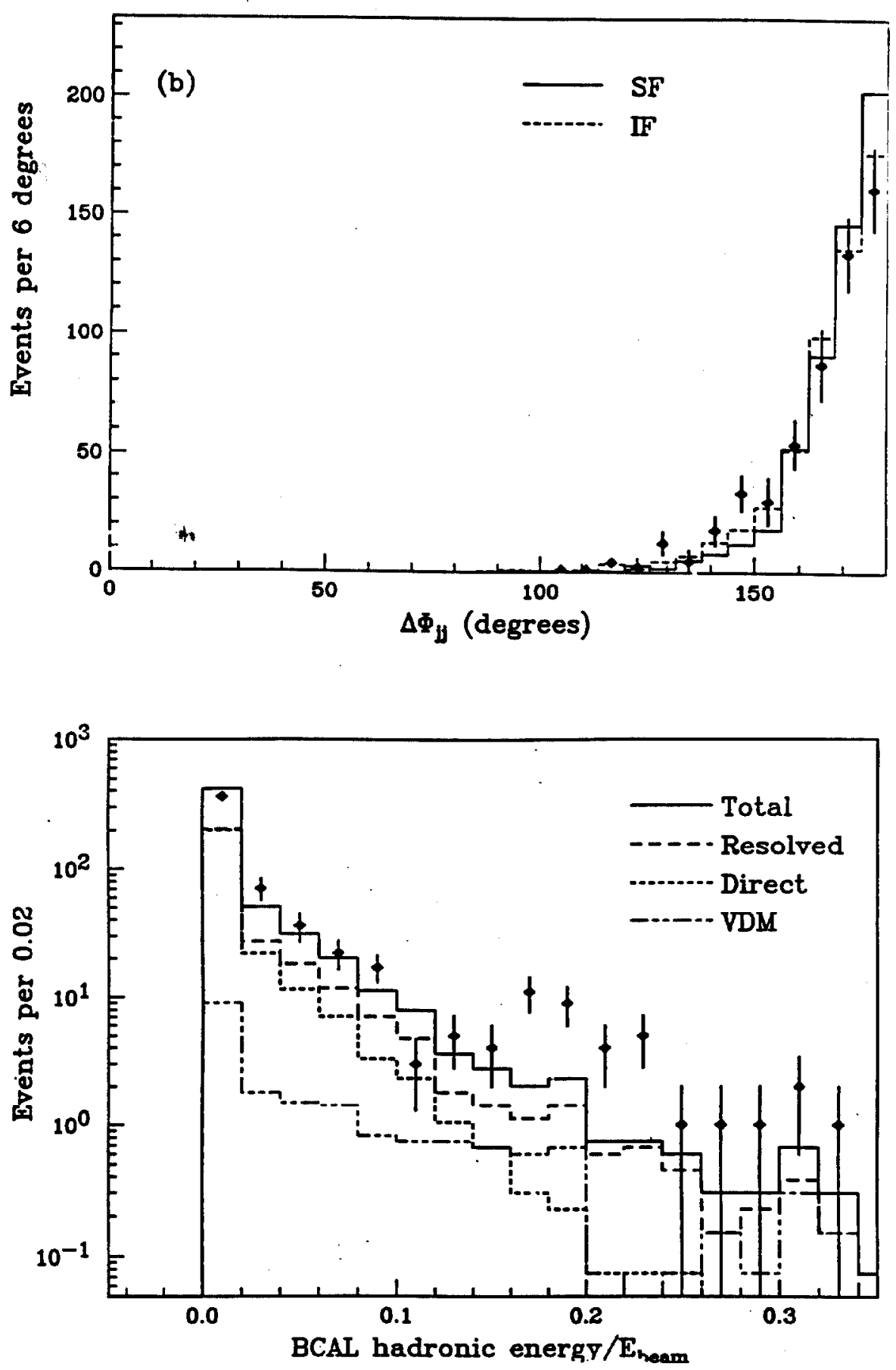

Figure 5: E683 measurements of a) the azimuthal separation between jets in photon interactions, b) the observed energy deposition in a small angle calorimeter compared with the various components of the photon interaction. 

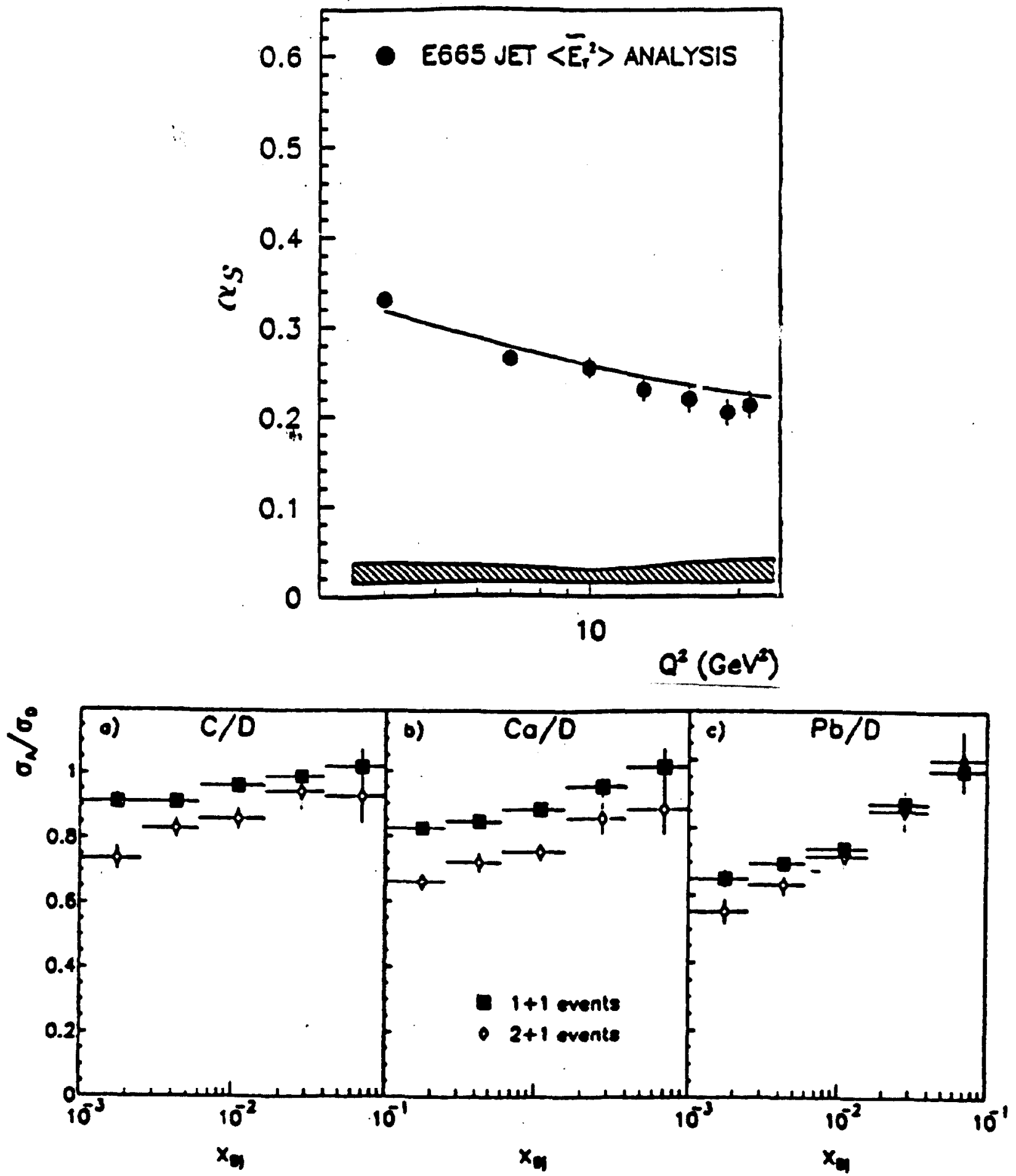

Figure 6: a) $\alpha_{S}$ as a function of $Q^{2}$ in muon scattering, b) the relative yields of $2+1$ and $1+1$ jet topologies in deep inelastic muon scattering, as a function of $x_{B j}$ from different nuclear targets. 

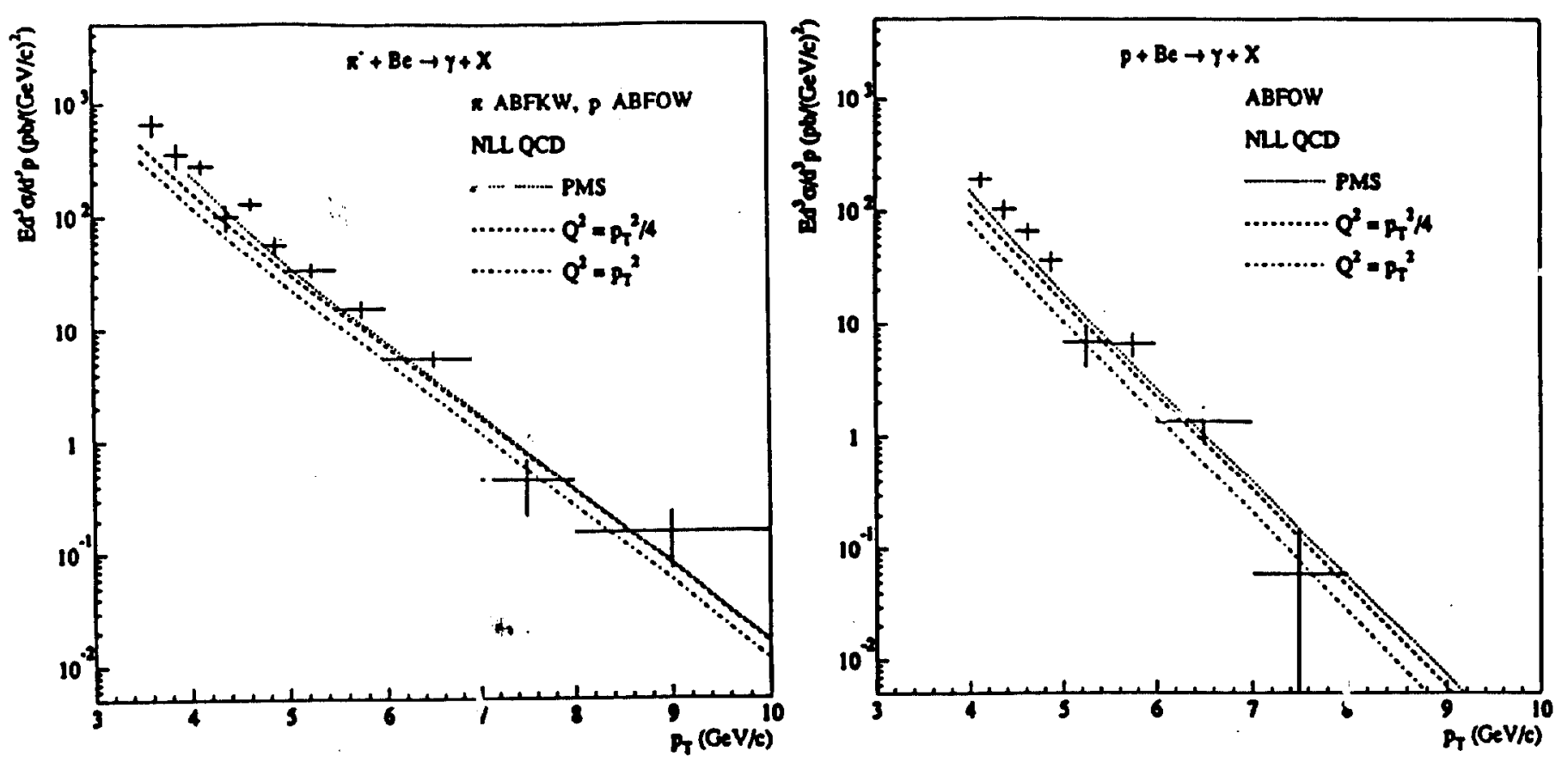

Figure 7: E706 single photon cross-sections compared with a variety of QCD calculations, a) for $\left.\pi^{-}, b\right)$ for protons.

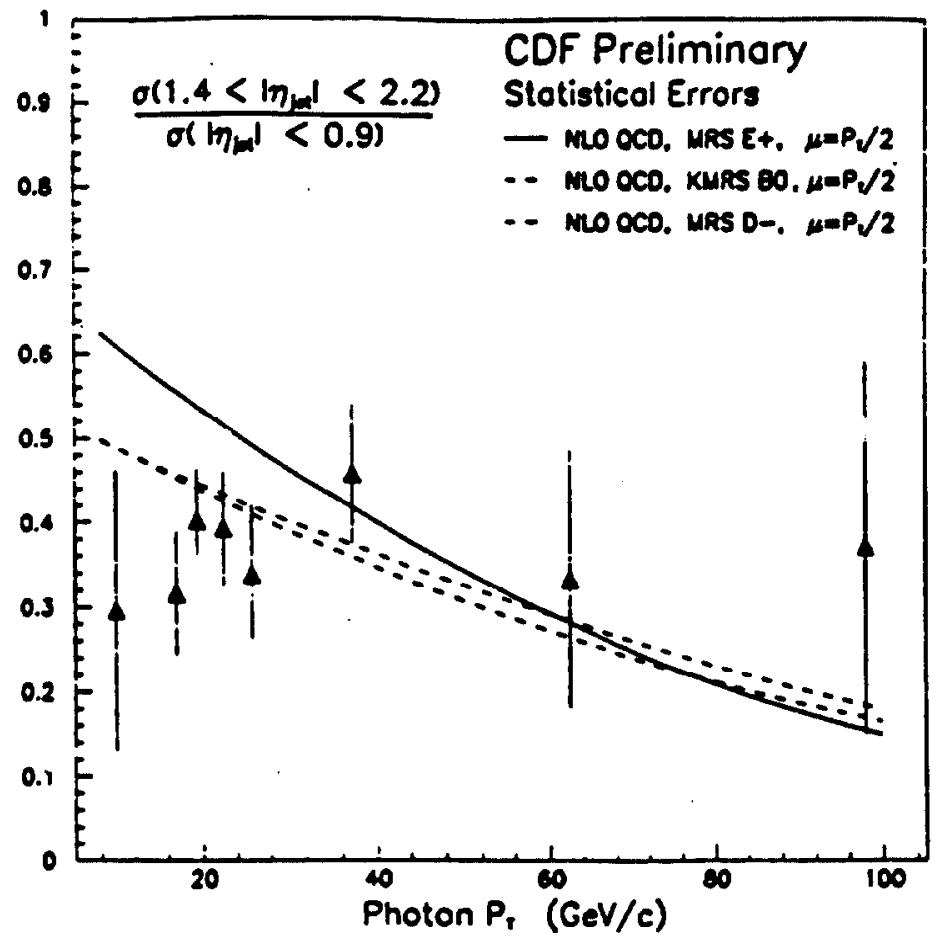

Figure 8: CDF, ratio of single photon cross-section in different $\eta$ ranges. 

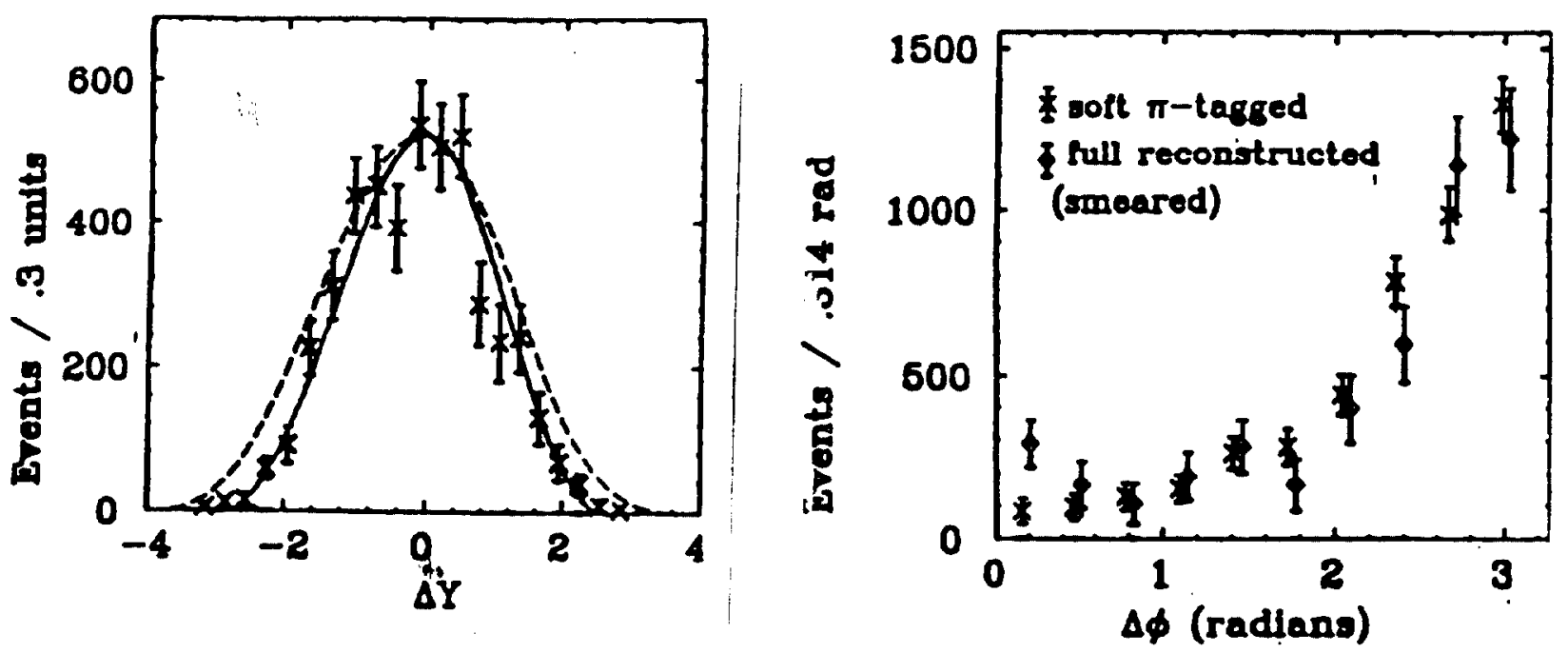

Figure 9: E687 measurements of charm pair production, a) the rapidity difference between pairs, b) the azimuthal angle difference between pairs.

The production cross-sections for mesons containing heavy quarks, charm and bottom, are expected to be amenable to perturbative QCD description. One of the generally accepted tests of this hypothesis is that the cross-section for production of charm mesons should be independent of atomic number, exhibiting no shadowing. Until recently there has been some inconsistency between different data sets. E769 [9] has studied the issue extensively. Their data shows no evidence for shadowing and now dominates the world data. The cross-section per nucleus is linear with atomic number at the few $\%$ level. The $x_{F}$ dependence shows[10] little difference when the produced meson contains quarks in common with the beam particle compared to when it does not. There is no evidence for a strong leading particle effect in contrast to earlier data. Recently there are measurements[11] which show modest differences when ratios of cross-sections are constructed as a function of $x_{F}$.

E687 has sufficient charm meson pairs to examine the production mechanism for photoproduction of charm and finds that the data[12] exhibit, see Fig. 9, the correlations between the charm pairs expected of the photon-gluon fusion mechanism. They use both fully reconstructed mesons and mesons in which a $D^{*}$ is tagged by a soft charged pion.

Within the last year, two fixed target experiments have reported cross-sections for bottom production. One, E672[14], bases the measurement on the rate of detached $J / \psi$ vertices. E653[13] used a hybrid emulsion spectrometer and attempted to fully reconstruct the bottom hadron decays. Based on nine pairs of bottom particles reconstructed they are able to look at 


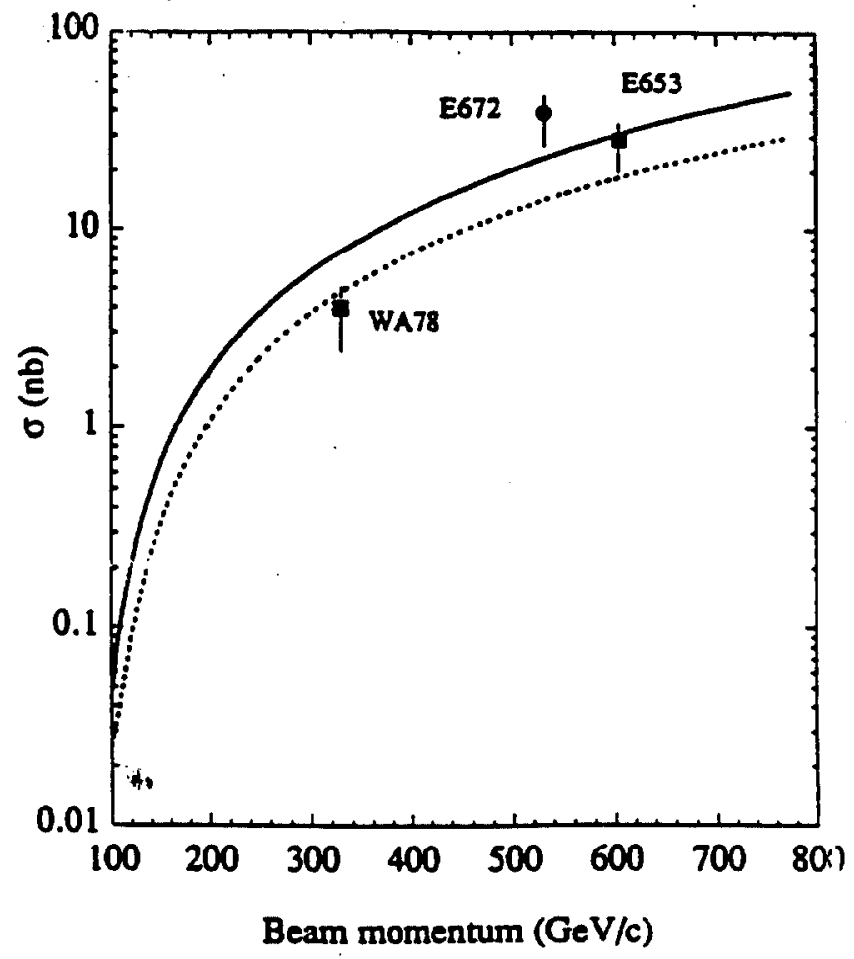

Figure 10: Bottom hadron production cross-section measurements at low energies.

the $x_{F}$ distribution as well as the cross-section. The results from both experiments are shown in Fig. 10 compared with the theoretical expectations.

In collider experiments the signal to background for heavy quark production is much more favorable. The semi-leptonic decays of charm mesons are characterized by a relatively low $p_{T}$ muon. As the $p_{T}$ rises, but the muon is still within a jet, bottom production becomes the dominant process. Fig 11 shows recent measurements[15] from $D \emptyset$ in which the different components of the single muon cross-section as predicted by theory are also shown. The agreement is good.

$\mathrm{CDF}$, with the silicon vertex detector and a magnetic field, are able to reconstruct, partially or fully, a limited number of bottom particles. They also deduce the bottom cross-section based on the yield of detached $J / \psi$ particles. The results[16] are shown in Fig. 12. The measurements appear to be high compared to the theoretical expectations. This is a possible area of disagreement between the two collider experiments which has not been resolved.

As mentioned earlier, there is a premium on measurements with a wide range of $\eta$ when the goal is to test QCD. $D \emptyset$ has a rather good calorimetric coverage and in Fig. 13, the jet cross-section[17] as a function of $p_{T}$ is shown for two different ranges out to $\eta=3.0$ and extending in $p_{T}$ to several hundred $\mathrm{GeV}$. This $\eta$ coverage is parlayed into a measurement of 

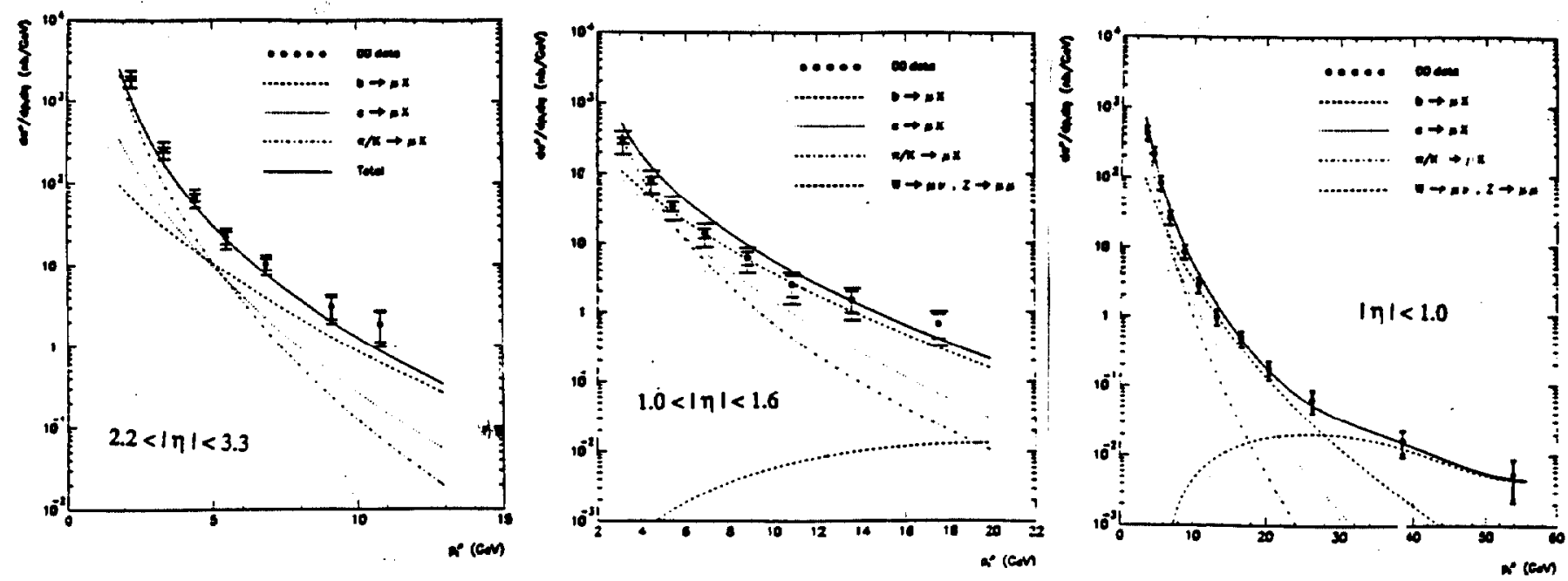

Figure 11: Measurement of the single muon cross-section at $1800 \mathrm{GeV}$ from DØ.

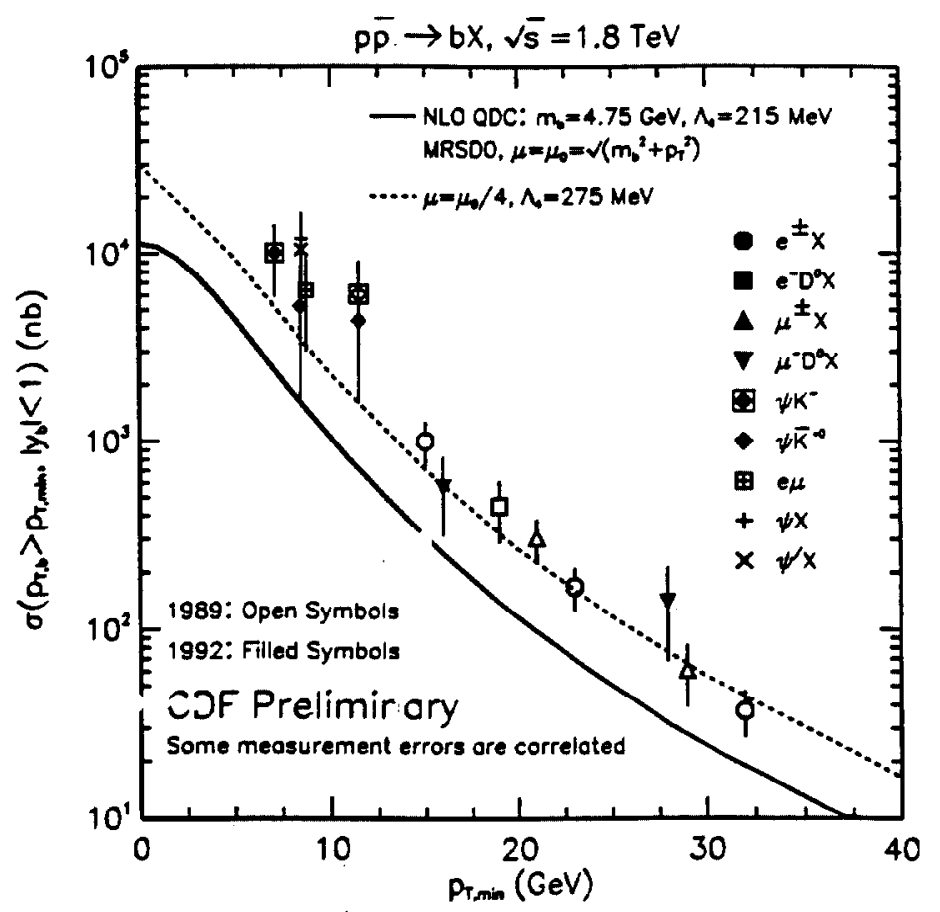

Figure 12: CDF B cross-section measurement. 

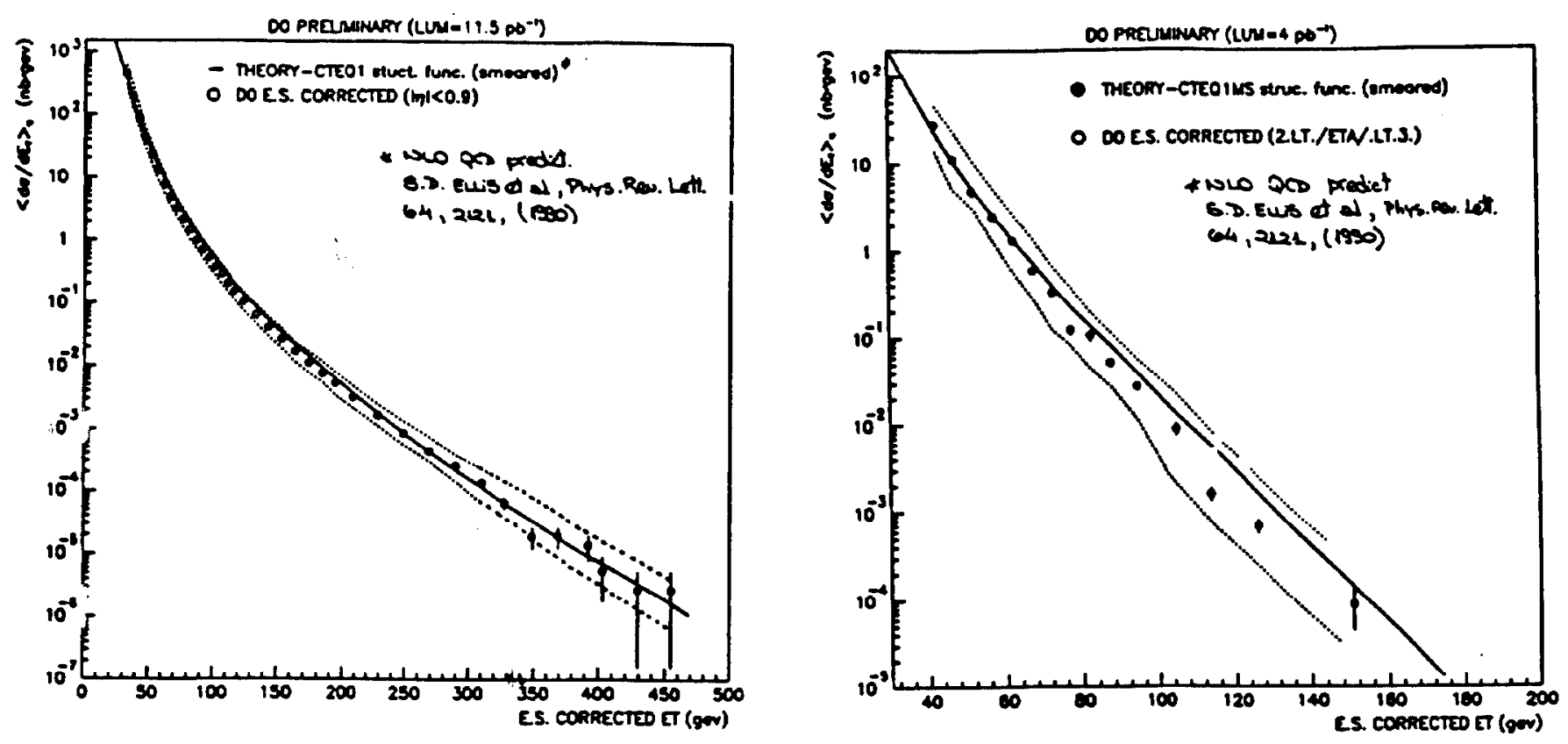

Figure 13: Jet production cross-sections in two different $\eta$ ranges from $\mathrm{D} \emptyset$.

the dijet angular distribution as shown in Fig. 14. If the underlying process were Rutherford scattering, the $\chi=\left(1+\cos \theta^{*}\right) /\left(1-\cos \theta^{*}\right)$, where $\theta^{*}$ is the parton center of mass scattering angle, distribution shown would be flat. Scale breaking in the parton distributions lead to the slope from low to high $\chi$. These data are a dramatic extension of the measured range.

Recently an old topic has been revitalised and new experimental results are appearing. In the 1970s rapidity gaps were a signal of the exchange of a pomeron. With the current theoretical understanding the more general case of the exchange of any colorless object has been considered[19] and a search has been executed[20] by $D \emptyset$. They find that if they plot the probability of finding nothing between two jets separated by some distance $\Delta \eta$ in pseudo-rapidity, then this probability decreases over a range $\Delta \eta \simeq 2$ and then reaches a plateau. As shown in Fig. 15, for $30 \mathrm{GeV}$ jets and where the threshold for something is defined by towers of 200 $\mathrm{MeV}$ in the electromagnetic calorimeter, the plateau probability is a few per thousand. The characteristic of the plot is very similar to that expected for a pomeron (a colorless combination of gluons) exchange. 


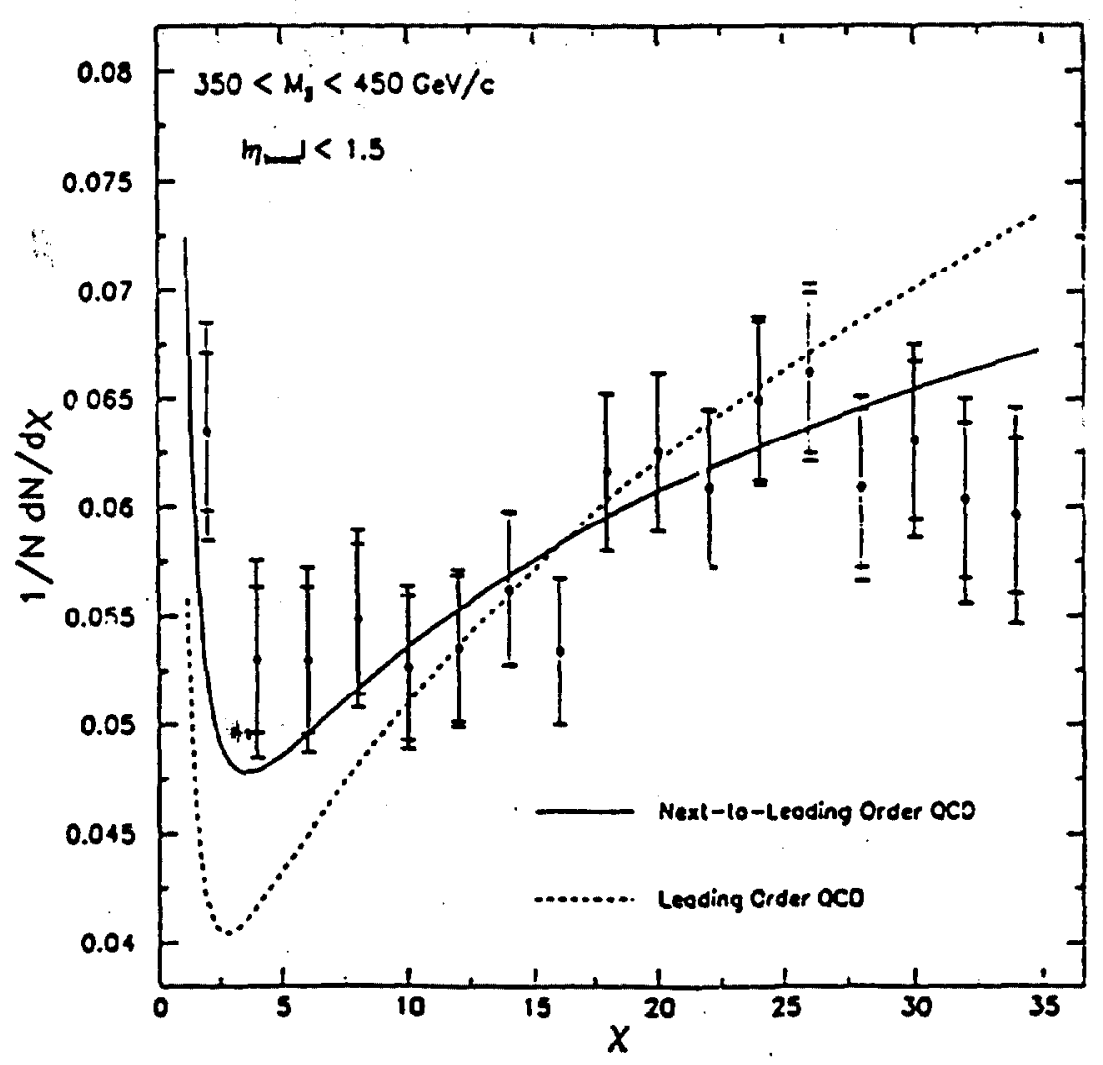

Figure 14: Dijet angular distributions.

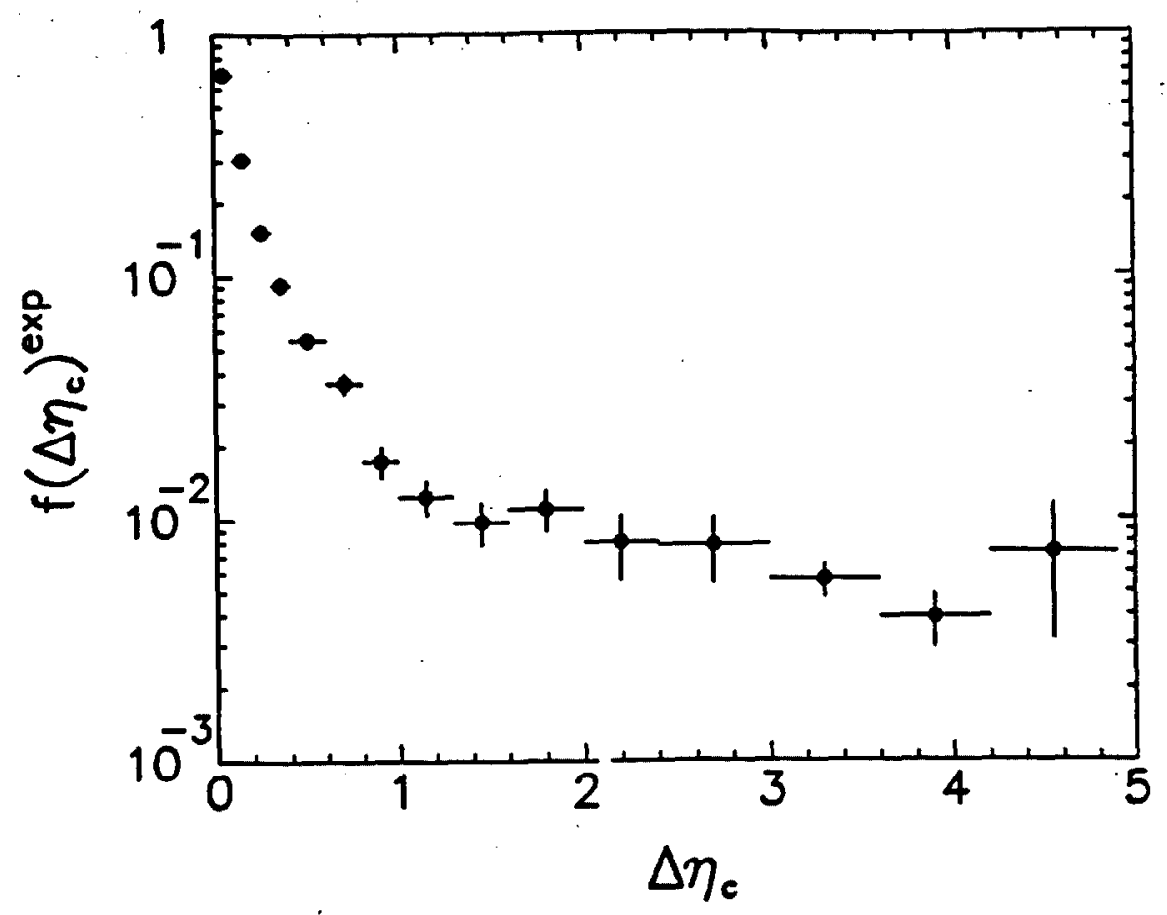

Figure 15: Fraction of events that have a tagged particle between the two leading jets as a fraction of their separation in pseudo-rapidity. 


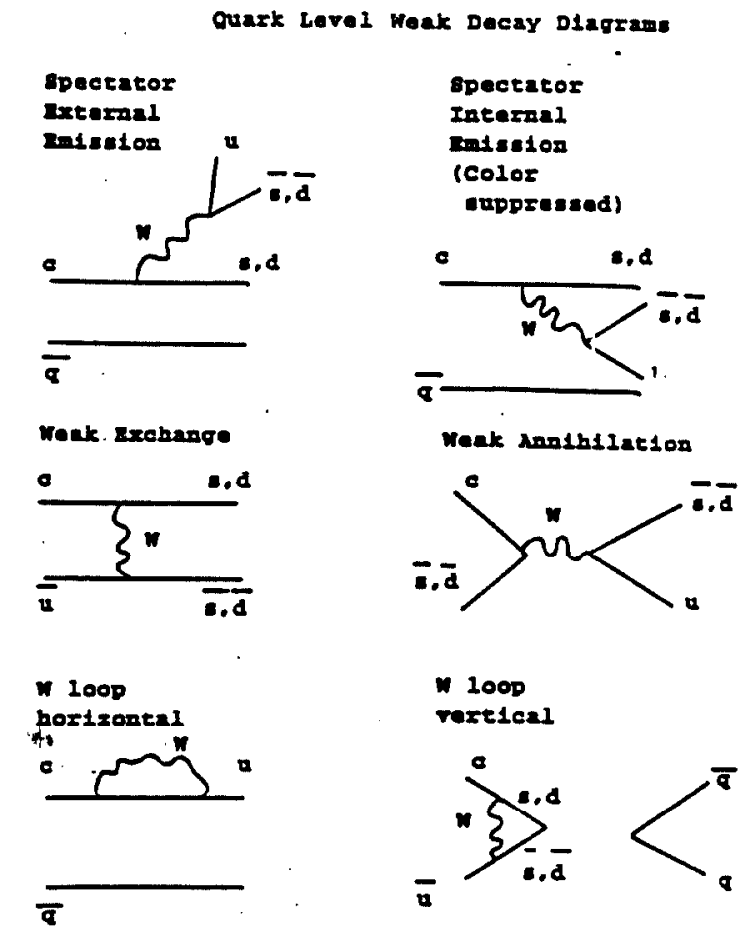

Figure 16: Quark level weak decay diagrams

\section{Decay Physics}

In the previous section we have referred to heavy quark production as a means of obtaining a handle on some of the more rigorous calculations of perturbative QCD. In the following we turn to the physics associated with the characteristics of the particular heavy flavor and its couplings to other flavors, the domain of the Cabibbo-Kobayashi-Maskawa(CKM) matrix. At least that will be the goal. As we shall see effects of the strong interaction are difficult to escape. Much of the content of this section comes from recent reviews[21][22].

First we consider charm decays, some relevant diagrams are shown in Fig. 16. The simplest are the spectator diagrams and the expectation is that as the mass of the heavy quark increases from strange to charm and to bottom, these diagrams should increasingly dominate. The understanding is that the semi-leptonic decays of the charmed mesons $D^{0}$ and $D^{+}$are equal but that complications in the hisizicic decays lead to an enhancement of those of the $\Sigma^{n}$ iy a factor 2 and hence make the $D^{0}$ lifetime correspondingly shorter. The $D_{a}^{+}$has a similar lifetime to that of the $D^{0}$. The data of Fig. 17, from E687, show the change in the fractions of $D^{+}$and $D_{s}^{+}$as the cut on the significance of the detachment of the decay vertex from the primary vertex is increased. The lifetime results for these cases suggest that the $\mathrm{W}$ exchange graph is not the dominant source of the charged-neutral difference. Rather, strong interaction interference effects are playing a big role. 

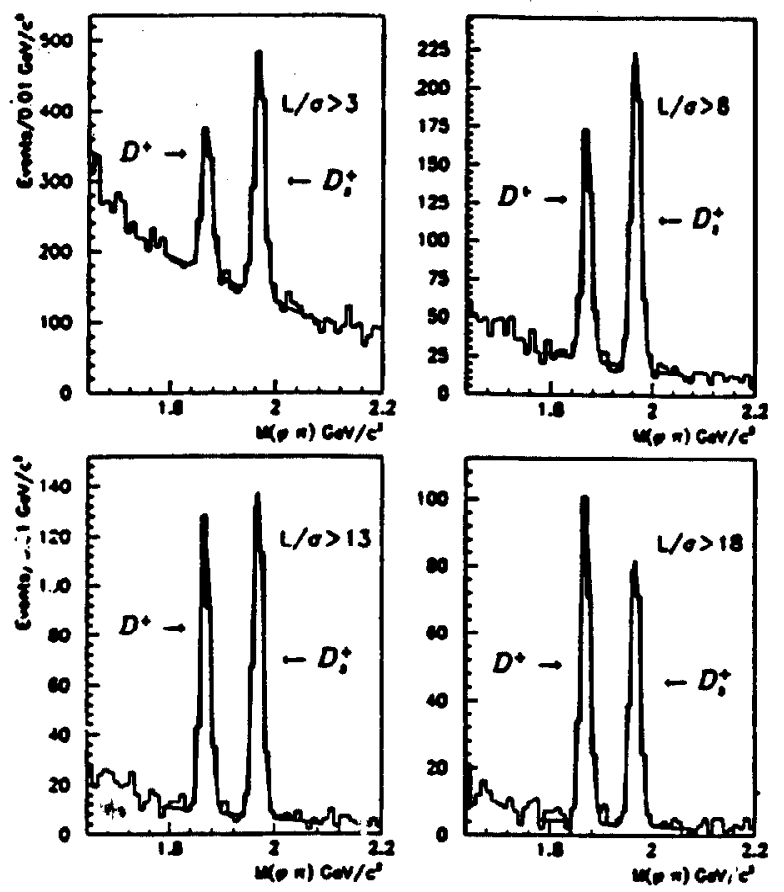

Figure 17: $D^{+}$and $D_{0}^{+} \rightarrow \phi \pi$ for increasing $L / \sigma$.

The charm baryons have lagged behind the charm mesons both in terms of number detected and in understanding. E687 are building an impressive array of results on this subject and one can compare the results for the lifetimes of the $\Lambda_{c}$, the $\Omega_{c}$ and the $\Xi_{c}$. The competing models[23][24] differ slightly in that the former would expect a hierarchy $\tau\left(\Omega_{c}\right)<\tau\left(\Xi^{0}{ }_{c}\right)<$ $\tau\left(\Lambda^{+}{ }_{c}\right) \simeq \tau\left(\Xi^{+}{ }_{c}\right)$ while the latter predicts $\tau\left(\Omega_{c}\right) \simeq \tau\left(\Xi_{c}^{0}\right)<\tau\left(\Lambda^{+} c\right)<\tau\left(\Xi_{c}^{+}\right)$. The data have $\tau\left(\Xi_{c}^{0}\right) / \tau\left(\Lambda_{c}^{+}\right)=0.47 \pm 0.12$ from E687, which favours the Guberina et al.[24] prediction at the level of $2 \sigma$ but which is not yet conclusive. Figure 18 shows the relevant mass peaks in the data from which the determinations are made. In contrast to the meson case, the $\mathrm{W}$ exchange is not expected to be helicity suppressed, and within the models, for some baryons, it plays a more significant role.

The $\mathrm{B}$ hadron lifetime has been measured by CDF[25] using their new silicon vertex detector to measure inclusive $J / \psi$ decay lengths. Figure 19 shows the proper length associated with the observed $J / \psi$ mass peak. It contains a component of prompt production with a symmetric distribution about zero. A background is determined from the sidebands, which is attributed to dimuons from the decay of two B mesons, or at least due to B meson decays, which results in a finite positive lifetime. There is also the real signal from $B \rightarrow J / \psi$. A combined fit, taking into account the scaling to the full $B$ meson momentum, results in the measurement $\tau(B)=1.46 \pm 0.06($ stat $) \pm 0.06($ syst $) p s$.

CDF have also detected a number of B meson exclusive decay modes involving the $J / \psi$ and by comparing $J / \psi K^{+}$with $J / \psi K^{* 0}$ they obtain a ratio between the lifetimes for charged 

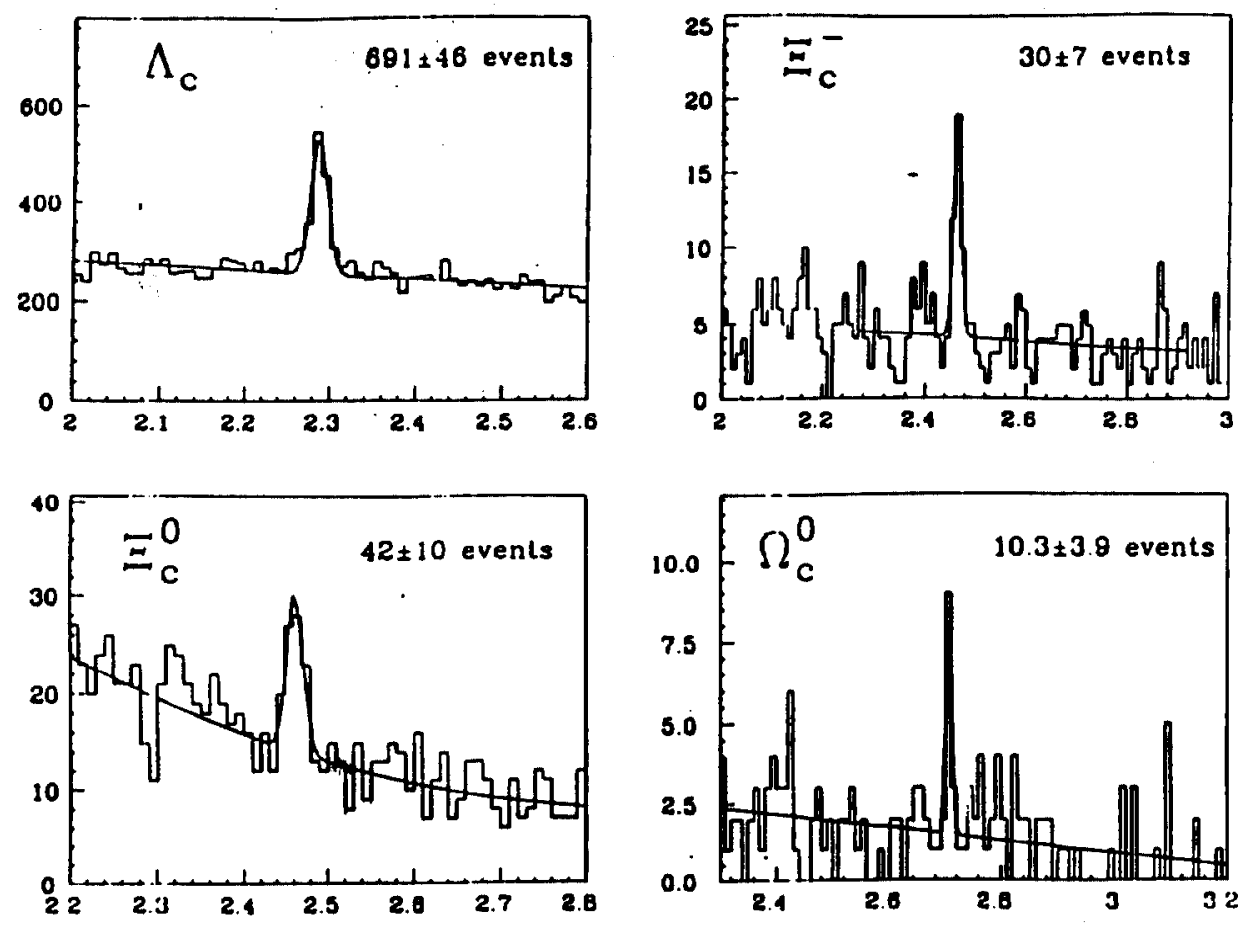

Figure 18: Charmed baryon mass peaks from E687.

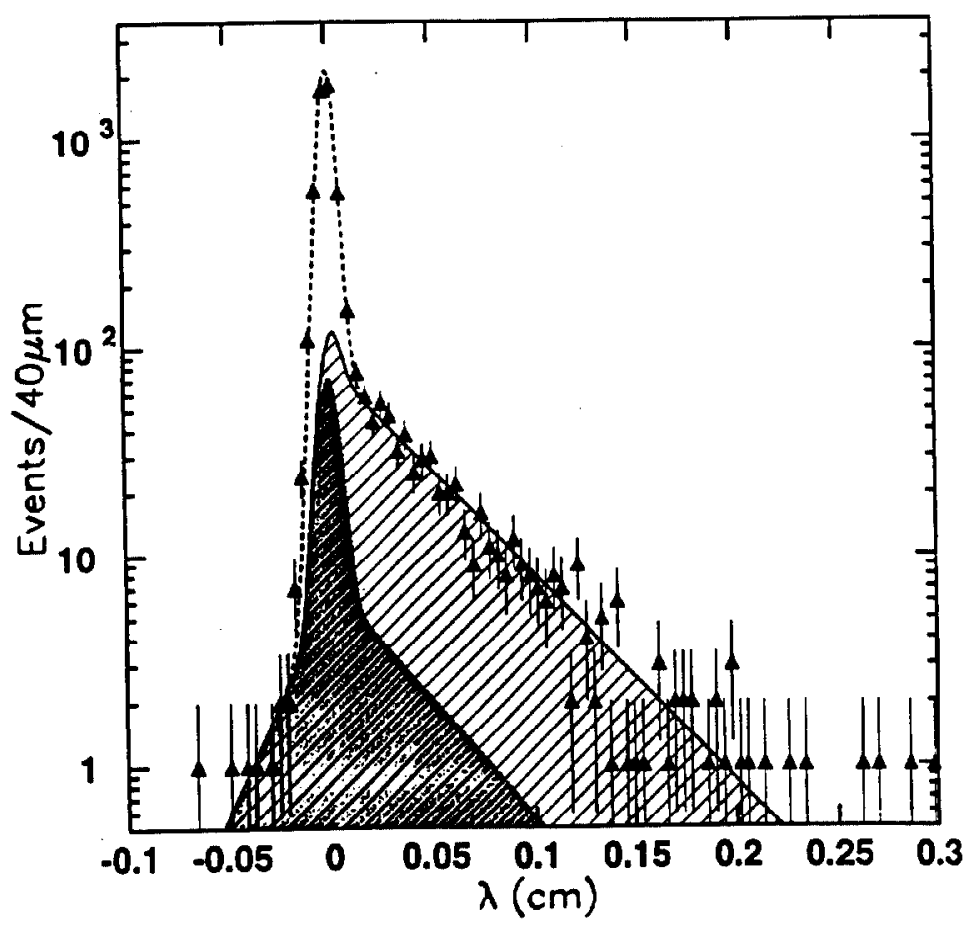

Figure 19: Proper decay length, $B \rightarrow J / \psi$. 


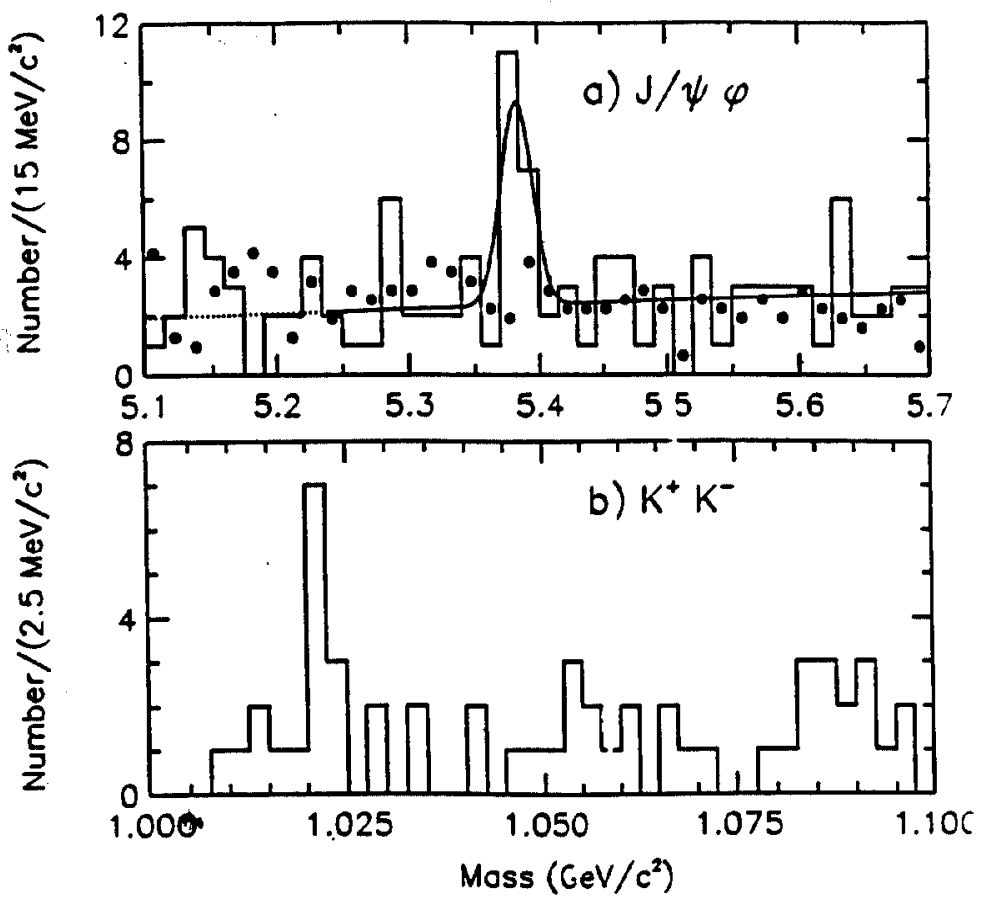

Figure 20: Mass peak for a) $B_{a}$ and b) $\phi$ in the decay.

and neutral $B$ mesons of $\tau\left(B^{+}\right) / \tau\left(B^{0}\right)=1.06 \pm 0.15$ (stat) \pm 0.05 (syst), this result is still preliminary. Finally, CDF has observed[26] the charmed strange meson through the decay $B_{a} \rightarrow J / \psi \phi$, the mass peak for the $B_{\text {a }}$ and for the $\phi$ are shown in Fig. 20. The mass value they quote[26] is $m_{B_{a}}=5383.3 \pm 4.5(s t a t) \pm 5.0(s y s t) M e V$ and the lifetime they obtain is $\tau\left(B_{\bullet}\right)=1.544_{-0.34}^{+0.42}($ stat $){ }_{-0.12}^{+0.11}(s y s t)$.

$B^{0}-\overline{B^{0}}$ mixing has been measured in several $e^{+} e^{-}$and $p \bar{p}$ experiments. The most recent measurement comes from $D \emptyset[27]$. They have examined the ratio of yields of like-sign and unlike-sign muons. The mass spectrum in the two cases is shown in Fig. 21. Cuts are applied to avoid the low mass region where the $J / \psi$ is evident in the unlike-sign spectrum. No isolation cuts are applied since the muons from the decays of B mesons are typically contained within the $b$ quark jets. The problem with the measurement is understanding the alternative sources of dimuons, for example sequential decays of the B mesons to charm which decay semi-leptonically. The result obtained after Monte Carlo correction for these and other effects is $\chi=0.13 \pm 0.02$ (stat) \pm 0.05 (syst), where $\chi$, the mixing parameter does not separate

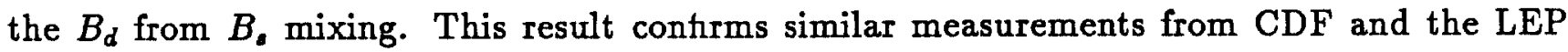
experiments with comparable precision.

Although there is hope for the B system, currently the only open window on CP violation is the $K^{0}-\overline{K^{0}}$ system. The produced states, $K^{0}$ and $\overline{K^{0}}$ are superpositions of the CP eigenstates $K_{1}^{0}$ and $K_{2}^{0}$. However, the states $K_{L}^{0}$ and $K_{S}^{0}$ observed through their decays appear to be themselves also superpositions of the $\mathrm{CP}$ eigenstates. The extent of the mixing is parameterised 

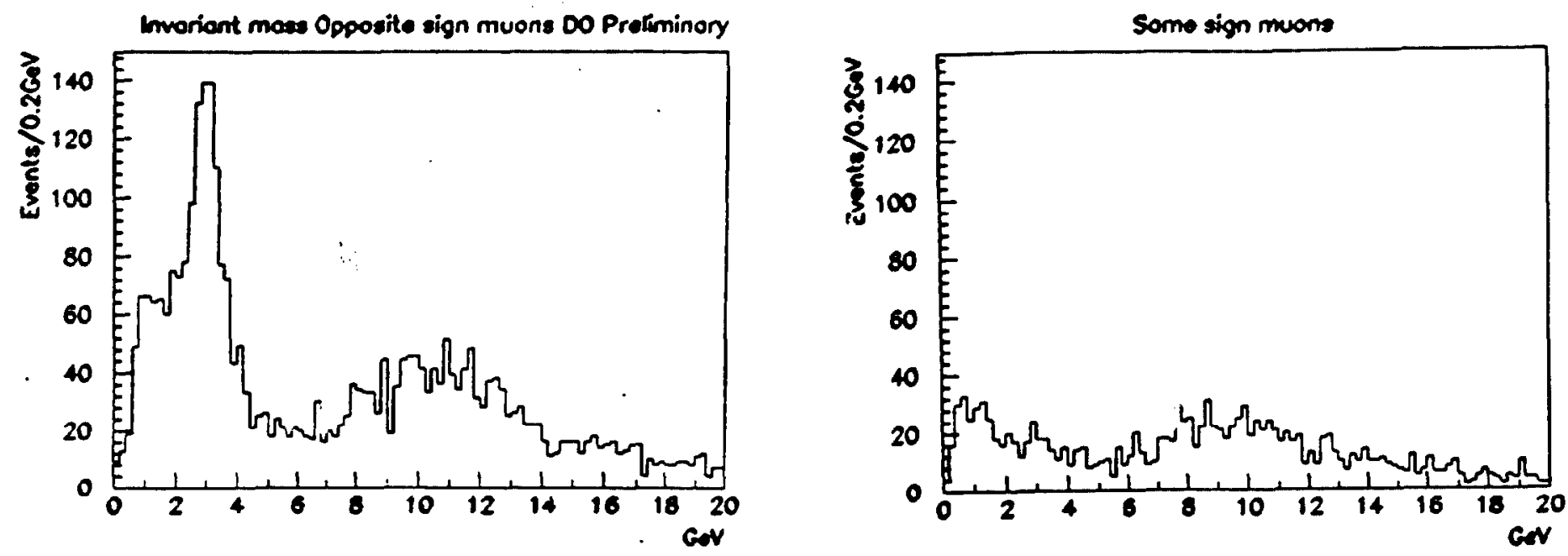

F gure 21: Mass spectra of Unlike-sign and Like-sign muc $n$ pairs.
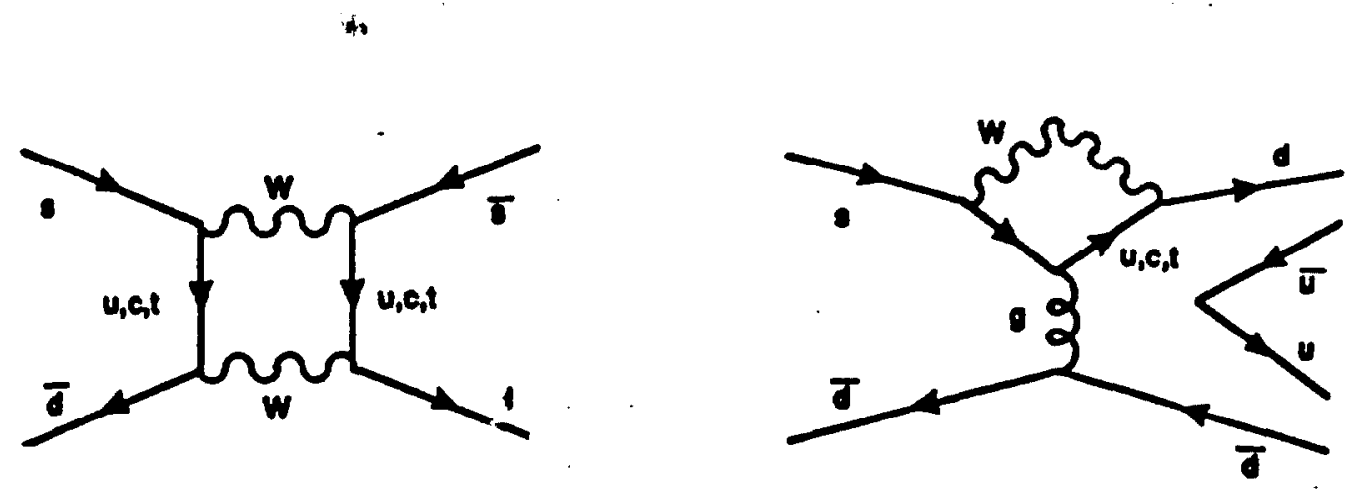

Figure 22: CP violating $K^{0}$ decay diagrams.

by the quantity $\epsilon$ which is determined by the inequalities of the decay rates of the $K_{L}^{0}$ and $K_{S}^{0}$ to two pions. Within the Standard Model this CP violation could have two origins depicted by the diagrams in Fig. 22a) and Fig 22b). The first, a box diagram containing a virtual top quark, would be a $\Delta S=2$ transition and is labeled indirect $C P$ violation; the second, a penguin diagram, would be $\Delta S=1$ and is termed direct $C P$ violation. The contribution of the direct $C P$ violation is measured by another parameter $\epsilon^{\prime}$. Experimentally its existence would lead to a difference between the ratio $K_{L}^{0} / K_{S}^{0}$ decaying to two charged pions as compared to two neutral pions. The Tevatron experiment E731 has recently published a result [28] of $\epsilon^{\prime} / \epsilon=(74 \pm 5.2($ stat $) \pm 2.9(s y s t)) 10^{-4}$. Other experiments, Ern? = 1d E773, use modifications of the E731 apparatus to look for CP violation in other channels and in the latter case to look for CPT violation. CPT studies are particularly interesting in the context of the theoretical lecture at this school by Nick Mavramatos[30][31]. The E773 measurement is of the phase difference between the charged and neutral two pion decays, currently the precision, from E731[32] is slightly more than 1 deg., the E773 measurement[29] will reduce that error by more than a factor two. 


\section{W/Z Physics}

The study of vector bosons is a primary domain for the $p \bar{p}$ collider and there are some new measurements from the Tevatron. New cross-section measurements have been presented by $D \emptyset[18]$ and $C D F[33]$ based on their 1992-93 data. The ratio of $W$ and $Z$ cross-section times branching ratio is an interesting quantity. It can be related through LEP measurements and some well established theore iral cross-section calculations, to the ratio of the lep onic and total widths of the $\mathrm{W}$ boson. In turn this ratio is sensitive to the existence of a low mass top quark. The measured values for the experimental ratios are CDF(electrons) $10.65 \pm$ 0.36 (stat) \pm 0.27 (syst), $\mathrm{CDF}$ (muons) $12.38 \pm 0.63$ (stat) \pm 0.45 (syst), D $\emptyset$ (electrons) $10.57 \pm$ 0.60 (stat) \pm 0.50 (syst) and DØ(mucns) $10.0 \pm 1.1$ (stat) $\pm 2.4(s y s t)$. For the CDF measurem.nt this translates into a decay mode independent top quark mass limit of $62 \mathrm{GeV}$ at $95 \%$ confidence level and for $\mathrm{D} \emptyset, 56 \mathrm{GeV}$.

The couplings between the vector bosons, the photon, $\mathrm{W}$ and $\mathrm{Z}$, are sensitive to the structure of the standard model and with the high energy of the Tevatron, there has been progress with the experimental measurements, particularly the measurement of the $\mathrm{W}-\gamma$ coupling. The signal for the events is a single photon in conjunction with the decay lepton from the $\mathrm{W}$ and the missing energy which is the signature of the partner neutrino. $D \emptyset[34]$ has shown results in both the electron and muon channels and finds limits on the anomalous magnetic moment of the W of $-2.5 \leq \Delta \kappa \leq 2.7$ at $95 \%$ confidence level. For the electric quadrupole moment the limits are $-1.2 \leq \lambda \leq 1.1$. These are significant improvements on the previous limits.

Finally, the primary goal of current $\mathrm{W}$ and $\mathrm{Z}$ physics is the precise measurement of the mass of the W. At the time of the Erice School there were no results from the 1992-93 data. Since that time both CDF and $D \emptyset$ have presented measurements and I give the status as of the Tsukuba $p \bar{p}$ Workshop. The measurement is very sensitive to the systematic errors associated with understanding the detector. Considerable effort is expended by both collaborations to understand their momentum scales whether they be those of magnetic tracking or those of the calorimetry. The result from $D \emptyset[35]$ is $M_{W}=79.86 \pm 0.16$ (stat) \pm 0.31 (syst) GeV \pm 0.30 (scale) $\mathrm{GeV}$ and from $\mathrm{CDF}[36] M_{W}=80.47 \pm 0.15($ stat $) \pm 0.25($ syst $) \mathrm{GeV}$. 


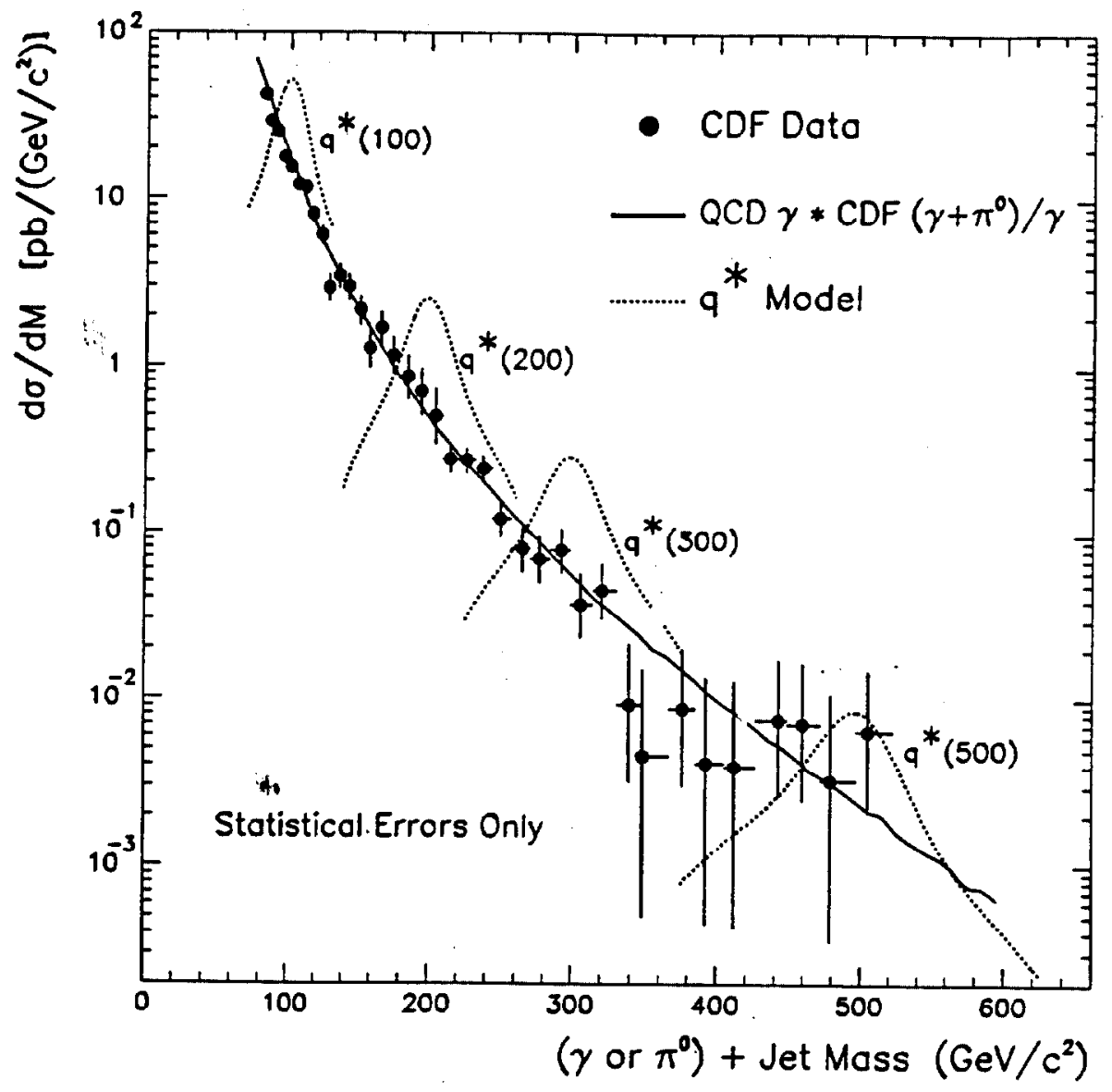

Figure 23: Excited quark production. a) shows the observed spectrum of quark-photon effective masses.

\section{Searches for New Physics}

In the category of searches for new physics we briefly consider searches for excited quarks and searches for leptoquarks, composites of leptons and quarks. Such searches are potentially the most important physics at the highest energy machine of the day. However, as yet all results are negative.

A first example[37] comes from the CDF group which has searched for excited quarks decaying to a quark, signalled by a jet, and a photon. In practice the latter objects may include some contamination from unresolved neutral pions. The results are shown in Fig. 23, where the observed yield is compared with that expected from background and putative resonance signals superimposed. When the background is subtracted, the result is compared to the theoretical expectations[38] in which the branching ratio for the decay is taken to be unity. In that case the result reduces to a mass limit of $470 \mathrm{GeV}$ at $95 \%$ confidence level for this and the analogous quark - boson possibility.

$D \emptyset$ has searched[39] for pair produced leptoquarks decaying into an electron and a quark 


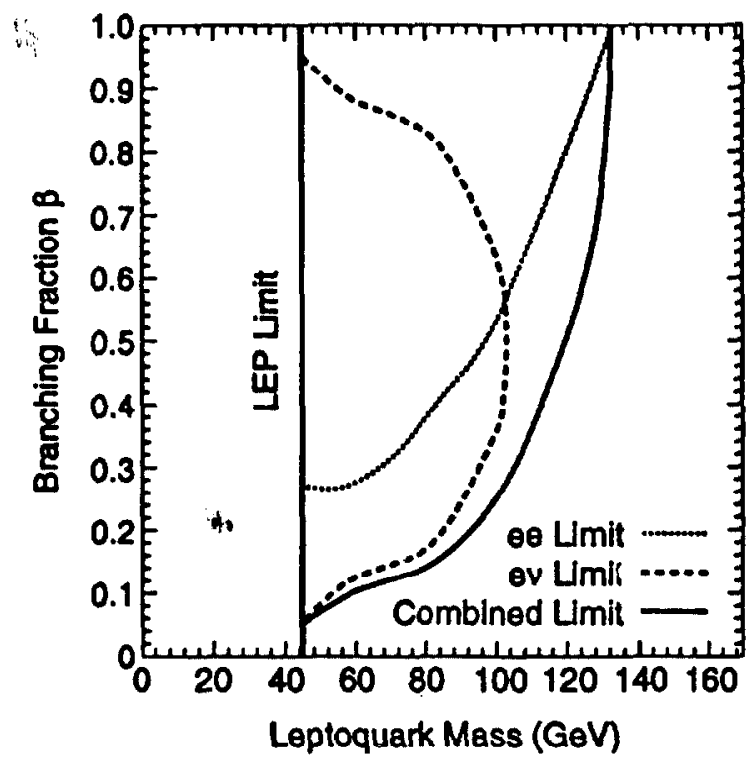

Figure 24: $\mathrm{D} \emptyset$ Lepto-quark mass limits as a function of branching ratio, $\beta$.

or a neutrino and a quark. The electron is detected in the calorimeter and the neutrino would result in significant missing transverse energy. The primary signals are then two electrons or one electron plus missing transverse energy. Since the particles, if they existed, would dominantly be pair produced at the Tevatron, such a method is relatively insensitive to the quark-leptonleptoquark coupling. The measurement leads to the limits shown in Fig. 24 for a range of decay branching ratios, $\beta$. Note that the use of both decay modes results in a limit which is about 3 times higher than the LEP limit except for values $\beta<0.2$. 


\section{The Search for the Top Quark}

The search for the top quark is a major activity within both of the Tevatron collider experiments, $C D F$ and $D \emptyset$. The analysis of the data is rapidly evolving. While the description given here follows that of the lecture, the quantitative limits are updated to correspond to the values shown by the two groups at the $p \bar{p}$ Workshop in Tsukuba in October, 1993,[40],[41],[42], [43].

Using data taken in 1989, CDF has shown[44] that it is unlikely that the mass of the top is less than that of the $\mathrm{W}$ boson. The discussion here therefore concentrates on the search for the top quarks where they are assumed to be produced in pairs $(t \bar{t})$ which then decay, each to a $\mathrm{W}$ boson and a $b$ quark. Schematically the possibilities are shown in Fig. 25. For the range of relatively high masses expected, the top quarks and their decay products populate the central range of rapidity, $|\eta|<2.5$. A potentially clean signal would be two high transverse momentum, isolated leptons $(e e, e \mu, \mu \mu)$ with associated missing transverse momentum and two hadronic jets. Although the branching ratio is low, this is a primary search channel. A signal of one high $p_{T}$ isolated lepton, missing transverse energy and four hadronic jets covers the case where one of the $\mathrm{W}$ bosons decays hadronically. This has a higher branching ratio but also has backgrounds associated with QCD W+ multi-jet production. In both experiments multiple triggers with different thresholds and combinations of requirements ensure relatively high efficiencies for the relevant modes.

The $\mathrm{D} \emptyset$ analysis of the dilepton channels concentrated on the $e e$ and $e \mu$ modes. For the $e e$ channel each electron had to have $p_{T}>20 \mathrm{GeV}$, and the missing $E_{T}$ had to be greater than $25 \mathrm{GeV}$, unless the dielectron mass was in the region of the $\mathrm{Z}$ peak when $40 \mathrm{GeV}$ of missing energy was required. Finally at least one other jet with $E_{T}>15 \mathrm{GeV}$ was required. One event was found. In the $e \mu$ analysis the analagous cuts were $15 \mathrm{GeV}$ in lepton $p_{T}$ and $20 \mathrm{GeV}$ in calorimeter missing $E_{T}$ and also the missing $E_{T}$ calculated taking into account the muon $p_{T}$. Separation of the electron and muon in pseudo-rapidity and azimuthal angle was also required and the muon required to be isolated, that is to say, not buried in a jet. Again one event was found.

Backgrounds were estimated using a combination of monte carlo studies and estimates for processes using ancillary data samples. The estimates of the backgrounds are about a half and one event in the $e e$ and $e \mu$ channels respectively. The expected $t \bar{t}$ yields are at the single event level for masses in the range 130 to $140 \mathrm{GeV}$. With these numbers no claim of discovery was made. The scatter plot of the muon $p_{T}$ versus the electron $p_{T}$ is shown in Fig. 26a) for data and in Fig 26b) for a large $160 \mathrm{GeV}$ top quark monte carlo sample. One sees that the event that 


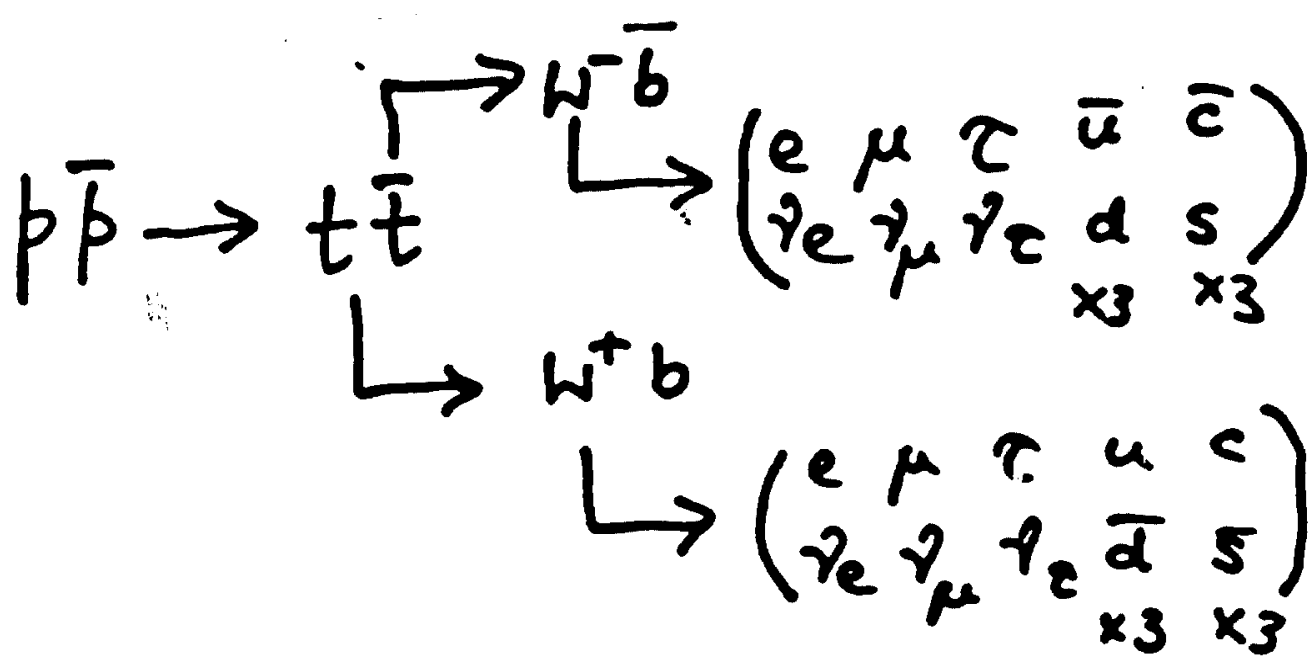

Figure 25: Top quark production and decay

the analysis cuts is, in fact, well above the cuts and it has generated considerable interest. Using the measurements of the leptons, the jets and the missing transverse momentum, the event is underconstrained. When the leptons and jets are organised in a particular combination and the $\mathrm{W}$ mass constraints and the requirement $m_{t}=\overline{m_{t}}$, are imposed, a kinematic fit is still not constrained. However, using knowledge of the incident parton distributions and the probabilities for different decay configurations, an analysis of the probability for different top masses can be performed[45]. Such an analysis, extended to account for detector resolutions and other effects, suggests a mass in the range around $145 \mathrm{GeV}$ [46] if this event is assumed to be from $t \bar{t}$.

As mentioned above the single lepton channels are expected to have significant background. The problem is that conventional $W$ boson production has higher order QCD contributions which lead to multiple jets which mock up the top quark signal. For this reason both CDF and $D \emptyset$ are examining more sophisticated approaches using either topological cuts or more promisingly, attempting to tag the $b$ jets which are expected to be more likely in the top events than in the $\mathrm{W}$ plus jets background. A $b$ jet has a good probability to contain a soft lepton from the semi-leptonic decay of either the $b$ or $c$ quarks in the chain. Also the decay length for the $b$ quark offers the possibility of tagging its presence by observing evidence for . üsplaced vertex. This is where the silicon vertex det:tsi in CDF plays a role. They have several vertex tagging methods and for the moment the details are unimportant. Fig. 27 shows the observed jet multiplicity distribution in the $\mathrm{W}$ plus jets (top candidate) events for a subsample of the data compared with the expectations from top of different masses. Even with the requirement of greater than 3 jets the data lie well above the signal expectations. If a vertex tagging requirement is applied, the situation shown in Fig 28 results. The data before the vertex tagging are replotted along with the data after applying the vertex tag. There are 

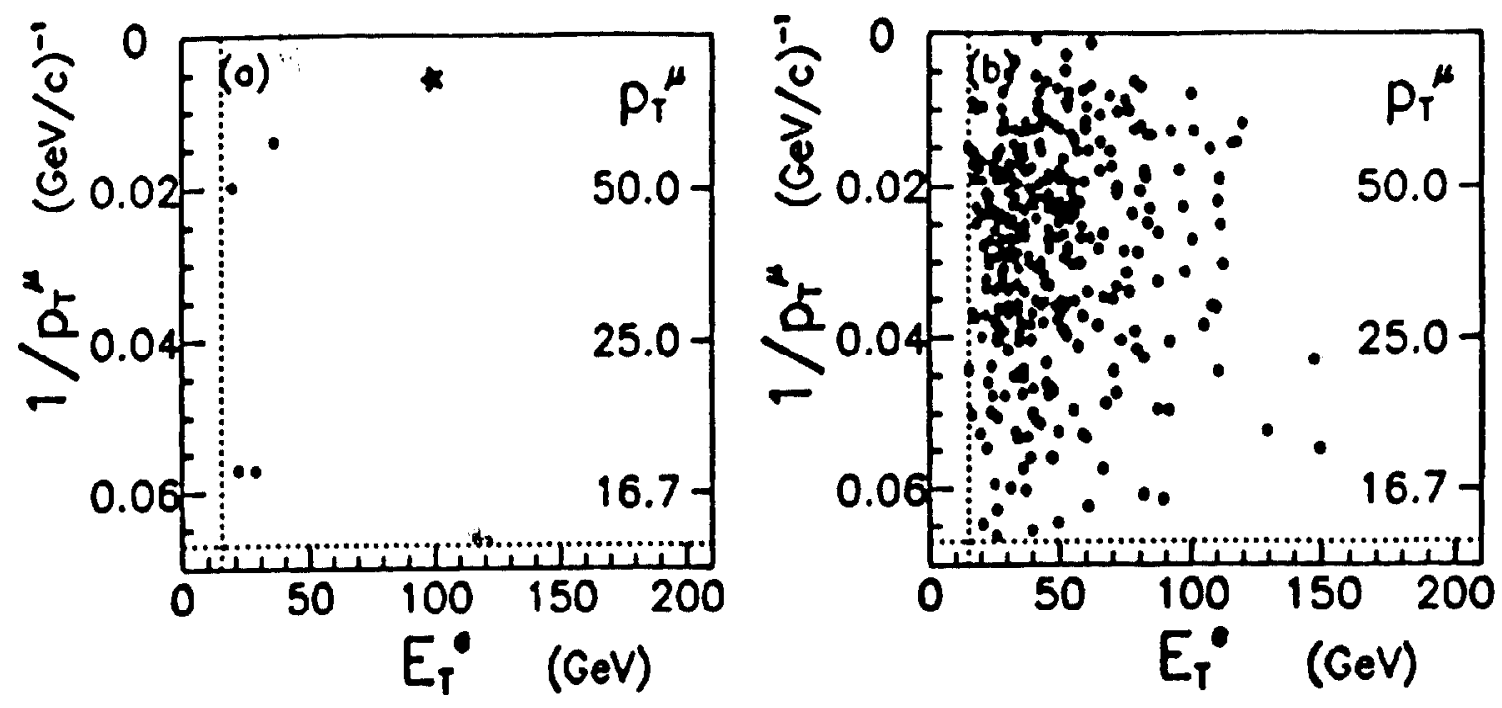

Figure 26: Scatter plot of lepton $p_{T}$ a) for the $e \mu$ data and b) for a top quark monte carlo with $m_{t}=160 \mathrm{GeV}$.

events with a jet multiplicity greater than three, two have a multiplicity of three. Further, the estimated background, as shown, is significantly less than one event. The analogous situation for a soft $(>4 \mathrm{GeV}$ ) muon tagging analysis is depicted in Fig 29. For a range of top masses the data, the background expectations and the signal expectations are all shown. With a greater than 3 jet requirement there appear to be no events in the data, a background expectation of about 0.2 events, and a signal plus background which is slightly greater than one event at $m_{t}=140 \mathrm{GeV}$. This analysis is fascinating but has not yet converged to the point where a statement is made about finding top nor to where the data are included in a limit estimation. It is worth repeating that these data represent about half of the total collected by CDF during the 1992-3 running. D $\varnothing$ using $a b$ tag analysis did include the lepton plus jets channel in a limit presented at Tsukuba[43]; however, here also the analysis has not yet matured to publication quality.

With the evolution in the analyses the situation with respect to the current quotable, $95 \%$ confidence limit on a lower mass limit is in flux and by the time this report appears, it may well have changed. Be that as it may, the situation was summarised at Tsukuba by Grannis[47]. He obtained $129 \mathrm{GeV}$ based on the channels presented by CDF and D $\emptyset$ at Cornell[48] and Tsukuba respectively. He used a NNLO cross-section calculation for the purpose. 


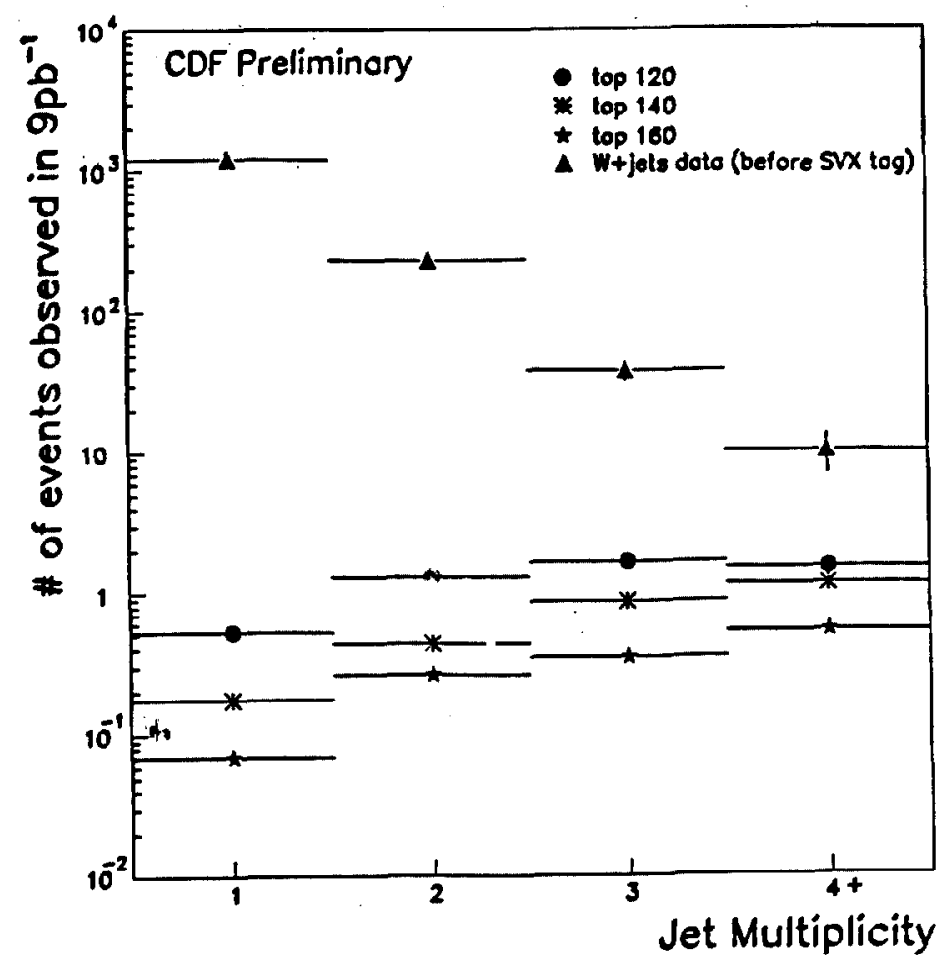

Figure 27: CDF lepton plus jets with no $\mathrm{b}$ tagging requirements.

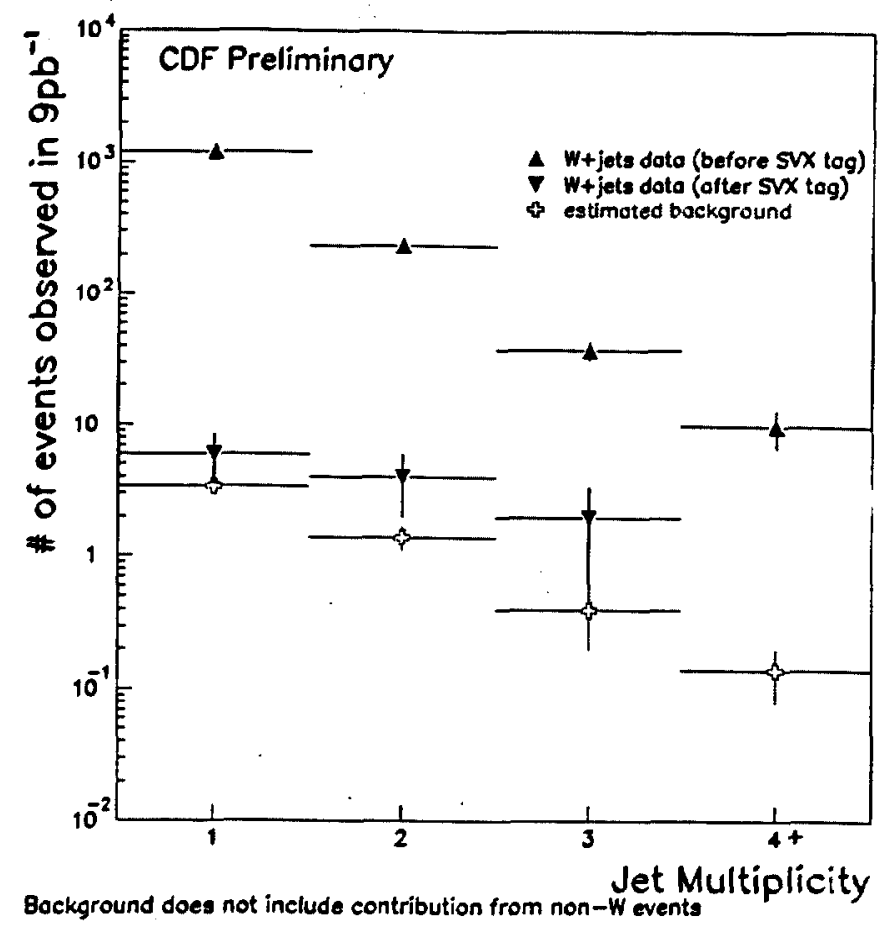

Figure 28: CDF lepton plus jets with detached vertex $\mathrm{b}$ tagging requirements. 


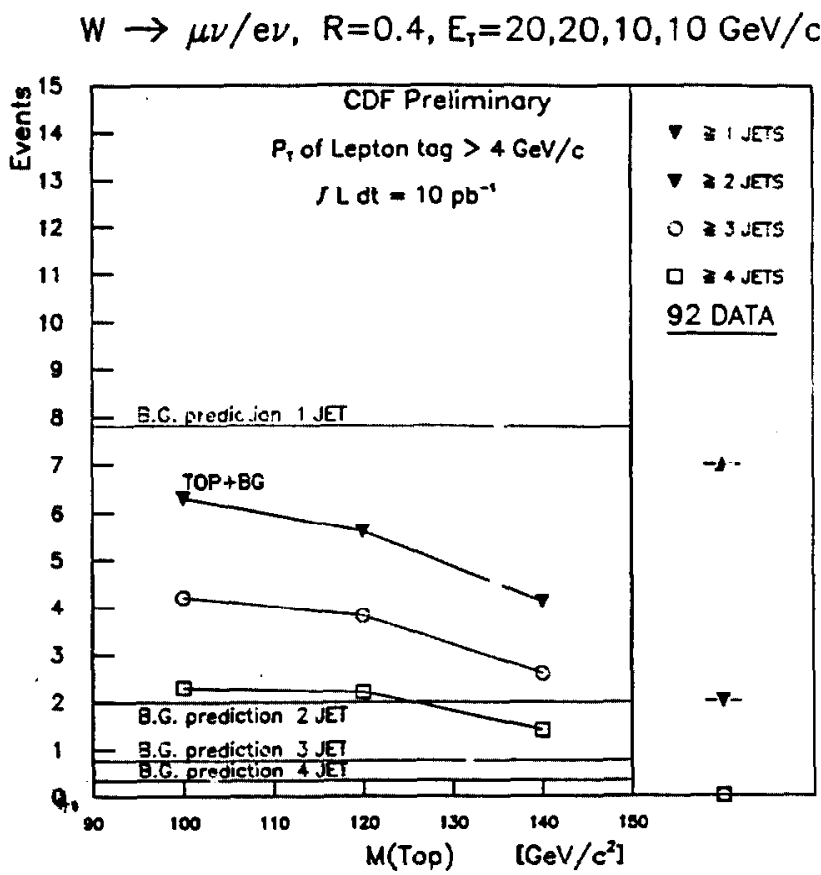

Figure 29: CDF lepton plus jets, soft muon b tagging requirements.

\section{Conclusions}

The aim of this talk was to give an impression of the tremendous range and depth of the data being produced by experiments at Fermilab, both fixed target and collider. Despite the generous allotment of time it was not possible to do more than scratch the surface of some subjects. The collider experiments, using the measurements of the $W$ mass and with the top search and mass limits, are approaching the situation where a statement about the Higgs mass, or a sensitive test of the consistency of the standard model become a possibility.

I would like to thank the Director of the Erice School and all its organizing staff for a very pleasant week and the students for the stimulating discourse. In addition, I would like to acknowledge the assistance of members of the Fermilab experiments who so generously gave their time and results. Finally, I would like to thank Sonya Wright for her assistance with the manuscript. 


\section{References}

[1] E760,T.Armstrong et al., Phys. Rev. Lett.68, 1468(1992).

[2] E683,D.Adams et al., Rice U. Preprint DE/F605/92ER40717-6, July 1993, FERMILABPub-94/025-E, submitted to Phys. Rev. Lett.

[3] E665,M.R.Adams et al., Phys . Rev. Lett. 72,466(1994).

[4] E665, M.R.Adams et al., FERMILAB-Pub-93/245-E, July 1993, submitted to Phys. Rev. D.

[5] H.Melanson, FERMILAB-Co.nf-93/165, presented at the 28th Rencontres de Morion 1, Les Arces, Savoie, France, March 20-27, 1993.

[6] E665, T.Carrol, FERMILAB-Conf-93/165, presented at the 28th Rencontres de Moriond, Les Arces, Savoie, France, March 20-27, 1993.

[7] G.Alverson et al., FERMILAB-Pub-93/007-E, January 1993, submitted to Phys. Rev. D.

[8] CDF,F.Abe et al., Phys. Rev. Lett. 71,679(1993); F.Abe et al., FERMILAB-Conf-93/206$\mathrm{E}$, Submitted to the 16th International Symposium on Lepton and Photon Interactions, Ithaca, Aug. 10-15, 1993.; F.Abe et al., FERMILAB-Conf-93/202-E, Submitted to the 16th International Symposium on Lepton and Photon Interactions, Ithaca, Aug. 10-15, 1993.

[9] E769, G.A.Alves et al., Phys. Rev. Lett. 70,722(1993).

[10] E769, G.A.Alves et al., FERMILAB-Pub-93/081-E, April 1993, submitted to Phys. Rev. Lett.

[11] J.Appel, XVI Int. Symp. on Lepton and Photon Interactions, Cornell U., Ithaca, New York, Aug. 1993.

[12] E687, P.L.Frabetti et al., Phys. Lett. B308, 193(1993).

[13] E653, K.Kodama et al., Phys. Lett. B305, 359(1993); K.Kodama et al., Prog. Theor. Phys., 89, 679(1993).

[14] E672, R.Jesik: $P h n$ Thesis, Univ. of Illinois at Chicago, August, 1993: R.Jesik et al., Proc. XXVI Int. Conf. on High Energy Physics, Dalas, Texas, 1992, AIP Conf. Proc. No 272, ed. J.R.Sandford, page 284.

[15] DØ, A. Maciel, Presented at the 9th Topical Workshop on $p \bar{p}$ Collider Physics, Tsukuba, Japan, October 17-22 1993.

[16] CDF, F.Abe et al., Phys. Rev. Lett. 71, 500(1993) F.Abe et al., FERMILAB-Pub-93/145 E, June 1993, submitted to Phys. Rev. Lett. 
[17] H.Weerts, Presented at the 9th Topical Workshop on $p \bar{p}$ Collider Physics, Tsukuba, Japan, October 17-22 1993.

[18] D $\emptyset$, N.Graf, Presented at the 9th Topical Workshop on $p \bar{p}$ Collider Physics, Tsukuba, Japan, October 17-22 1993.

[19] J.D.Bjorken, Phys. Rev. D47, 101(1992).

[20] D $\varnothing$, F.Borcherding, Presented at the 9th Topical Workshop on $p \bar{p}$ Collider Physics, Tsukuba, Japan, October 17-22 1993. S.Abachi et al., FNAL-Pub-94/005-E, 1994, to be published in Phys. Rev. Lett.

[21] P.Garbincius, Invited Talk pres:nted at Hadron '93 Conference, Centro di Cultura scientifica "A.Volta", Villa Olmo, Como, Italy, June 21-25,1993 .

H.

[22] Joel Butler and Peter H.Garbincius, Fermilab Report, Summer, 1993.

[23] M.B.Voloshin and M.A.Shifman, Sov. Phys, JETP 64,698(1987).

[24] B.Guberina,R.Ruckl and J.Trampetic, Z. Phys. C33, 297(1986).

[25] CDF, F.Abe et al., FERMILAB-Pub-93/158-E, June 1993, submitted to Phys. Rev. Lett.

[26] CDF,F.Abe et al.,Phys. Rev. Lett. 71,1685(1993).

[27] D $\emptyset$, S.Igarashi, Presented at the 9th Topical Workshop on $p \bar{p}$ Collider Physics, Tsukuba, Japan, October 17-22 1993.

[28] E731, L.K. Gibbons, Phys. Rev. Lett. 70, 1203 (1993).

[29] E773, G. Gollin, Private Communication.

[30] Talk by Nick Mavramatos at this school.

[31] The relevance of the measurement to CPT violation was discussed by M.Gourdin in the discussion session associated with this lecture at this school.

[32] E731, L.K.Gibbons, Phys. Rev. Lett. 70, 1199 (1993).

[33] CDF, W. Badgett, Presented at the 9th Topical Workshop on $p \bar{p}$ Collider Physics, T'sukuba, Japan, October 17-22 1993.

[34] Dø, A.Spadafora, Presented at the 9th Topical Workshop on $p \bar{p}$ Collider Physics, Tsukuba, Japan, October 17-22 1993.

[35] Dø, Q. Zhu, Presented at the 9th Topical Workshop on $p \bar{p}$ Collider Physics, Tsukuba, Japan, October 17-22 1993. 
[36] CDF, D.Saltzberg, Presented at the 9th Topical Workshop on $p \bar{p}$ Collider Physics, Tsukuba, Japan, October 17-22 1993.

[37] CDF, FERMILAB-Pub-93/341-E, Nov. 1993, Submitted to Physical Review Letters.

[38] U.Baur, I.Hinchliffe and D.Zeppenfeld, Int. Journal of Mod. Phys. A2(1987)1285.

[39] Dø, S. Abachi et al, FERMILAB-Pub-93/340-E, 1993, to be published in Phys. Rev Lett.

[40] CDF, T.Chikamatsu, Presented at the 9th Topical Workshop on $p \bar{p}$ Collider Physics, Tsukuba, Japan, October 17-22 1993.

[41] CDF, M.Contreras, Presented at the nth Topical Workshop on $p \bar{p}$ Collider Physics, Tsukuba, Japan, October 17-22 1993.

[42] D $\emptyset$, M.Fatyga, Presented at the 9th Topical Workshop on $p \bar{p}$ Collider Physics, Tsukuba, Japan, October 17-22 1993.

[43] D $\varnothing$, H.Greenlee, Presented at the 9th Topical Workshop on $p \bar{p}$ Collider Physics, Tsukuba, Japan, October 17-22 1993.

[44] CDF, F.Abe, et al., Phys. Rev. D 45, 3921 (1992).

[45] R.H.Dalitz and G.R.Goldstein, Phys. Lett. B287, 225 (1992); K.Kondo, T.Chikamatsu and S.Kim, Journal of the Phys. Soc. of Japan, 62,1177(1993).

[46] D $\emptyset$, M.Strovink, Proceedings of the International Europhysics Conference on High Energy Physics, Marseille, France, 1993, eds. J.Carr and M.Perrottet.

[47] P.Grannis, Presented at the 9th Topical Workshop on $p \bar{p}$ Collider Physics, Tsukuba, Japan, October 17-22 1993.

[48] P.Tipton, XVI Int. Symp. on Lepton and Photon Interactions, Cornell U., Ithaca, New York, Aug. 1993. 